\title{
Hypernuclear weak decay puzzle
}

\author{
C. Barbero, ${ }^{1,4}$ D. Horvat, ${ }^{2}$ F. Krmpotić, ${ }^{1}$ T. T. S. Kuo, ${ }^{5}$ Z. Narančić, ${ }^{2}$ and D. Tadić ${ }^{3}$ \\ ${ }^{1}$ Departamento de Física, Universidad Nacional de La Plata, C. C. 67, 1900 La Plata, Argentina \\ ${ }^{2}$ Department of Physics, Faculty of Electrical Engineering, University of Zagreb, 10000 Zagreb, Croatia \\ ${ }^{3}$ Physics Department, University of Zagreb, 10000 Zagreb, Croatia \\ ${ }^{4}$ Centro Brasileiro de Pesquisas Físicas, Rua Dr. Xavier Sigaud 150, 22290-180 Rio de Janeiro-RJ, Brazil \\ ${ }^{5}$ Department of Physics, SUNY-Stony Brook, Stony Brook, New York 11794
}

(Received 22 August 2001; revised manuscript received 23 July 2002; published 27 November 2002)

\begin{abstract}
A general shell model formalism for the nonmesonic weak decay of the hypernuclei has been developed. It involves a partial wave expansion of the emitted nucleon waves, preserves naturally the antisymmetrization between the escaping particles and the residual core, and contains as a particular case the weak $\Lambda$-core coupling formalism. The extreme particle-hole model and the quasiparticle Tamm-Dancoff approximation are explicitly worked out. It is shown that the nuclear structure manifests itself basically through the Pauli principle, and a very simple expression is derived for the neutron- and proton-induced decays rates $\Gamma_{n}$ and $\Gamma_{p}$, which does not involve the spectroscopic factors. We use the standard strangeness-changing weak $\Lambda N \rightarrow N N$ transition potential which comprises the exchange of the complete pseudoscalar and vector meson octets $\left(\pi, \eta, K, \rho, \omega, K^{*}\right)$, taking into account some important parity-violating transition operators that are systematically omitted in the literature. The interplay between different mesons in the decay of ${ }_{\Lambda}^{12} C$ is carefully analyzed. With the commonly used parametrization in the one-meson-exchange model (OMEM), the calculated rate $\Gamma_{\mathrm{NM}}=\Gamma_{n}+\Gamma_{p}$ is of the order of the free $\Lambda$ decay rate $\Gamma^{0}\left(\Gamma_{\mathrm{NM}}^{\mathrm{th}} \cong \Gamma^{0}\right)$ and is consistent with experiments. Yet the measurements of $\Gamma_{n / p}=\Gamma_{n} / \Gamma_{p}$ and of $\Gamma_{p}$ are not well accounted for by the theory $\left(\Gamma_{n / p}^{\text {th }} \lesssim 0.42, \Gamma_{p}^{\text {th }}\right.$ $\left.\gtrsim 0.60 \Gamma^{0}\right)$. It is suggested that, unless additional degrees of freedom are incorporated, the OMEM parameters should be radically modified.
\end{abstract}

DOI: 10.1103/PhysRevC.66.055209

PACS number(s): 21.80.+a, 21.60.-n, 13.75.Ev, 25.80.Pw

\section{INTRODUCTION}

The free $\Lambda$ hyperon weak decay (with transition rate $\Gamma^{0}$ $=2.50 \times 10^{-6} \mathrm{eV}$ ) is radically modified in the nuclear environment. First, as a result of the Pauli principle, the mesonic decay rate $\Gamma_{M} \equiv \Gamma(\Lambda \rightarrow N \pi)$ is strongly blocked for $A \geqslant 4$. Second, new nonmesonic (NM) decay channels $\Lambda N \rightarrow N N$ become open, where there are no pions in the final state. The corresponding transition rates can be stimulated either by protons, $\Gamma_{p} \equiv \Gamma(\Lambda p \rightarrow n p)$, or by neutrons, $\Gamma_{n} \equiv \Gamma(\Lambda n$ $\rightarrow n n)$. The ultimate result is that in the mass region above $A=12$ the total hypernuclear weak decay rates $\Gamma_{M}+\Gamma_{\mathrm{NM}}$ $\left(\Gamma_{\mathrm{NM}}=\Gamma_{n}+\Gamma_{p}\right)$ are almost constant and close to $\Gamma^{0}[1]$.

Because of the practical impossibility of having stable $\Lambda$ beams, the NM decays in hypernuclei offer the best opportunity to examine the $\Delta S=-1$ nonleptonic weak interaction between hadrons. Yet the major motivation for studying these processes stems from the inability of the present theories to account for the measurements, in spite of the huge theoretical effort that has been invested in this issue over several decades [2-26]. More precisely, the theoretical models reproduce fairly well the experimental values of the total width $\Gamma_{\mathrm{NM}}\left(\Gamma_{\mathrm{NM}}^{\exp } \cong \Gamma^{0}\right)$, but the ratio $\Gamma_{n / p} \equiv \Gamma_{n} / \Gamma_{p} \quad(0.5$ $\leqslant \Gamma_{n / p}^{\exp } \leqslant 2$ ) remains a puzzle.

In the one-meson-exchange model (OMEM), which is very often used to describe the hypernuclear $\Lambda N \rightarrow N N$ decay, it is assumed that the process is triggered via the exchange of a virtual meson. The obvious candidate is the onepion-exchange (OPE) mechanism, and following the pioneering investigations of Adams [3], ${ }^{1}$ several calculations have been done in ${ }_{\Lambda}^{12} C$, yielding $\Gamma_{\mathrm{NM}}^{(\mathrm{OPE})} \cong \Gamma^{0}$ and $\Gamma_{n / p}^{(\mathrm{OPE})}$ $\cong 0.1-0.2[10,12,13,21,24]$.

The importance of the $\rho$ meson in the weak decay mechanism was first discussed by McKellar and Gibson [4]. They found that, because of the sensitivity of the results to the unknown $\Lambda N \rho$ vertex, the estimates for $\Gamma_{\mathrm{NM}}$ could vary by a factor of 2 or 3 when the potential $V_{\rho}$ was included. (See also Ref. [5].) The present-day consensus is, however, that the effect of the $\rho$ meson on both $\Gamma_{\mathrm{NM}}$ and $\Gamma_{n / p}$ is small $[10,12,13,15]$.

Until recently, there have been quite dissimilar opinions regarding the full OMEM, which encompasses all pseudoscalar mesons $(\pi, \eta, K)$ and all vector mesons $\left(\rho, \omega, K^{*}\right)$. In fact, while Dubach et al. [12] claimed that the inclusion of additional exchanges in the $\pi+\rho$ model plays a major role in increasing the $n / p$ ratio, Parreño, Ramos, and Bennhold [13] and Sasaki, Inoue, and Oka [21] argued that the overall effect of the heavier mesons on this observable was very small. However, the two latter groups have recently corrected their calculations for a mistake in including the $K$ and $K^{*}$ mesons, and so their estimates of $\Gamma_{n / p}$ have been augmented quite substantially $[22,24]$. Almost simultaneously, Oset, Jido, and Palomar [25] have also shown that the $K$ meson contribution was essential to increase $\Gamma_{n / p}$. However, the experimental data have not been fully explained yet.

\footnotetext{
${ }^{1}$ McKellar and Gibson [4] have pointed out that this publication contains a very important error and that the decay rates given by Adams [3] should be multiplied by 6.81 .
} 
In the last few years, many other attempts have not been particularly successful either in accounting for the measured $\Gamma_{n / p}$ ratio. To mention just a few of them: (1) analysis of the two-nucleon stimulated process $\Lambda N N \rightarrow N N N[7,9,14]$, (2) inclusion of interaction terms that violate the isospin $\Delta T=1 / 2$ rule $[16,20]$, (3) description of the short-range baryon-baryon interaction in terms of quark degrees of freedom [17,21], and (4) introduction of correlated two-pion exchange potentials besides the OPE [18]. Consistent (though not sufficient) increases of the $n / p$ ratio were found in the last two works. (For instance, $\Gamma_{n / p}$ was boosted up to 0.36 for the decay of ${ }_{\Lambda}^{12} C[18]$.) In fact, only Jun [26] was able so far to reproduce well the $\Gamma_{\mathrm{NM}}, \Gamma_{p}$, and $\Gamma_{n / p}$ data. He has employed, in addition to the OPE, an entirely phenomenological four-baryon point interaction for short-range interactions, including the $\Delta T=3 / 2$ contribution as well, and has conveniently fixed the different model coupling constants. Let us also note that after the present work had been completed, Itonaga et al. [27] have updated their studies and have performed extensive calculations of the NM decays in the mass region $4 \leqslant A \leqslant 209$, which have revealed that the correlated- $2 \pi$ and $-1 \omega$ exchange potentials significantly improve the $\Gamma_{n / p}$ ratios over the OPE results.

In the OMEM, a weak baryon-baryon-meson (BBM) coupling is always combined with a strong BBM coupling. The strong one is determined experimentally with some help from the $\mathrm{SU}(3)$ symmetry, and the involving uncertainties have been copiously discussed in the literature [28-31]. It is the weak BBM couplings which could become the largest source of errors. In fact, only the weak $N \Lambda \pi$ amplitude can be taken from the experiment, at the expense of neglecting the off-mass-shell corrections. All other weak BBM couplings are derived theoretically by using $\mathrm{SU}(3)$ and $\mathrm{SU}(6)_{w}$ symmetries, octet dominance, current algebra, partial conservation of axial vector current (PCAC), pole dominance, etc. $[6,12,13,32-37]$. Assortments of such methods have been developed and employed for a long time in weak interaction physics to explain the hyperon nonleptonic decays. Specifically, to obtain the weak BBM couplings for vector mesons the $\mathrm{SU}(6)_{w}$ symmetry is used, which is not so well established as the $\mathrm{SU}(3)$ symmetry is. Moreover, the results derived by way of the $\mathrm{SU}(6)_{w}$ symmetry depend on the contributions of factorizable terms $a_{V}$ and $a_{T}$, which were only very roughly estimated [12,32,34]. Well aware of all these limitations, McKellar and Gibson [4] have allowed for an arbitrary phase between the $\rho$ and $\pi$ amplitudes in the $\pi$ $+\rho$ model. The same criterion was adopted by Takeuchi, Takaki, and Bando [5].

We wish to restate that the OMEM transition potential is purely phenomenological and that it is not derived from a fundamental underlying form, as happens, for instance, in the case of electromagnetic transitions or semileptonic weak decays. Only the OPE model is a natural and simple extrapolation of the mesonic decay mechanism of the $\Lambda$ to the NM process: the weak BBM coupling is identical to that used in the phenomenological description of the free $\Lambda$, and the strong vertex is the one traditionally used in describing the $\pi N N$ vertex. The assumption is that this is a valid approximation, although the pion is off the mass shell. Accordingly, all modern interpretations of the NM weak decay use the OPE as the basic building block for the medium- and longrange part of the decay interaction. On the contrary, the full OMEM is not used very often and, in place of the one-meson $\eta, K, \ldots$ exchanges, other mechanisms are employed as referred to above. One should also keep in mind that both the strong and weak BBM couplings, as well the meson masses, can become significantly renormalized by the nuclear environment [38].

The high momentum transfer in the NM decay makes the corresponding transition amplitude very sensitive to the short-range behavior of the $N N$ and $N \Lambda$ interactions. In fact, quite recently it has been pointed out that the final state interactions (FSIs) have a very large influence on the total and partial decay rates [24] (see also Ref. [27]). ${ }^{2}$ As a result of the same reason, one could expect that the nuclear structure effects not included in the main field [such as the random phase approximation (RPA) or pairing correlations, higher-order seniority excitations in the initial and final states, etc.] should not play an important role. Yet it could be useful to understand this issue more genuinely and to get a more complete control on the nuclear structure aspect of the problem. These are the main motivations for the present work.

The only existing shell model framework for the hypernuclear decay is the one based on the weak coupling model (WCM) between the hyperon and the $(A-1)$ core $[8,13,18,27]$. It involves the technique of coefficients of fractional parentage, and the spectroscopic factors (SFs) explicitly appear in the expressions for the transition rates. Yet in nuclear structure calculations it is in general simpler to evaluate the transition probabilities directly from the wave functions, instead of doing it via the SFs. Here we first develop a fully general shell model formalism and then we work out thoroughly the extreme particle-hole model (EPHM) and quasiparticle Tamm-Dancoff approximation (QTDA) for the even-mass hypernuclei.

Owing to the above-mentioned characteristics of the OMEM, it might be legitimate to ask whether it is possible to account for all three data $\Gamma_{\mathrm{NM}}, \Gamma_{p}$, and $\Gamma_{n / p}$ by not fully complying with the constraints imposed by the SU(3), $\mathrm{SU}(6)_{w}$, and chiral symmetries on the BBM couplings. To find out in which way these parameters should be varied we perform a multipole expansion of the transition rate in the framework of the EPHM, which unravels in an analytic way the interplay between different mesons in each multipole channel.

Attention will be given also to the parity-violating potential, since there are several typographical errors in the recent papers $[13,19,24]$, regarding this part of the transition potential. We will also consider some important contributions due

\footnotetext{
${ }^{2}$ The FSIs also make hard the extraction of the $n / p$ ratio from the experimental data [39-43], and to surmount this difficulty Hashimoto et al. [43] have quite recently combined the Monte Carlo FSI internuclear cascade models from Ref. [14] with the geometry of the detectors. Moreover, Golak et al. [47] have shown that the FSIs, in principle, hinder the measurement of the $n / p$ ratio in ${ }_{\Lambda}^{3} H$.
} 
to the vector mesons, which, although always included in the description of the nuclear parity violation [32,44-46], have been so far neglected in all studies of the NM hypernuclear decays, except those of Dubach et al. [12,34].

The outline of this paper is as follows: The general shell model formalism for the hypernuclear $\Lambda N \rightarrow N N$ weak decay is developed in Sec. II. The nonrelativistic approximation for the effective Hamiltonian is presented in Sec. III. The EPHM and QTDA are explained in Sec. IV, where the multipole expansion of $\Gamma_{\mathrm{NM}}$ is also done. Numerical evaluations of the ${ }_{\Lambda}^{12} C \rightarrow{ }^{10} C+n n$ and ${ }_{\Lambda}^{12} C \rightarrow{ }^{10} B+p n$ decay rates are carried out in Sec. V, and the conclusions are presented in Sec. VI. The formulas for the nuclear matrix elements are summarized in the Appendix.

\section{TRANSITION RATE}

The decay rate, of a hypernucleus (with spin $J_{I}$ and en$\operatorname{ergy} \mathcal{E}_{I}$ ) to residual nuclei (with spins $J_{F}$ and energies $\mathcal{E}_{F}$ ) and two free nucleons (with total spin $S$ and energies $\epsilon_{p}$ and $\left.\epsilon_{P}\right)$, follows from Fermi's golden rule:

$$
\begin{aligned}
\Gamma= & 2 \pi \sum_{S M_{S} J_{F} M_{F}} \int\left|\left\langle\mathbf{p P S} M_{S} ; J_{F} M_{F}|V| J_{I} M_{I}\right\rangle\right|^{2} \\
& \times \delta\left(\epsilon_{p}+\epsilon_{P}+\mathcal{E}_{F}-\mathcal{E}_{I}\right) \frac{d \mathbf{p}}{(2 \pi)^{3}} \frac{d \mathbf{P}}{(2 \pi)^{3}} .
\end{aligned}
$$

Here $V$ is the weak hypernuclear potential, the wave functions for the kets $\left|\mathbf{p P} S M_{S} ; J_{F} M_{F}\right\rangle$ and $\left|J_{I} M_{I}\right\rangle$ are assumed to be antisymmetrized and normalized, and a transformation to the relative and center-of-mass (c.m.) momenta $\mathbf{p}$ and $\mathbf{P}$ is already implied, i.e.,

$$
\mathbf{p}=\frac{1}{2}\left(\mathbf{p}_{1}-\mathbf{p}_{2}\right), \quad \mathbf{P}=\mathbf{p}_{1}+\mathbf{p}_{2}
$$

It is convenient to define the quantity

$$
\begin{aligned}
\mathcal{I}(p, P)= & (4 \pi)^{-4} \sum_{S M_{S} J_{F} M_{F}} \int d \Omega_{p} d \Omega_{P} \\
& \times\left|\left\langle\mathbf{p P} S M_{S} ; J_{F} M_{F}|V| J_{I} M_{I}\right\rangle\right|^{2},
\end{aligned}
$$

and rewrite Eq. (2.1) as

$$
\Gamma=\frac{16 M_{N}^{3}}{\pi} \int_{0}^{\Delta_{F}} d \epsilon \sqrt{\epsilon\left(\Delta_{F}-\epsilon\right)} \mathcal{I}(p, P),
$$

where $P=2 \sqrt{M_{N} \epsilon}, p=\sqrt{M_{N}(\Delta-\epsilon)}, \Delta_{F}=\mathcal{E}_{I}-\mathcal{E}_{F}-2 M_{N}$, and $M_{N}$ is the nucleon mass.

The partial wave expansion of the wave function of the nonantisymmetrized two-particle ket $\left.\mid \mathbf{P p} S M_{S}\right)$ is then performed:

$$
\begin{aligned}
\left(\mathbf{r R} s_{1} s_{2} \mid \mathbf{P} \mathbf{p} S M_{S}\right)= & (4 \pi)^{2} \sum_{l m L M} i^{l+L} Y_{l m}^{*}(\hat{p}) Y_{L M}^{*} \\
& \times\left(\hat{P}\left(\mathbf{r R} s_{1} s_{2} \mid p l m, P L M, S M_{S}\right),\right.
\end{aligned}
$$

where

$$
\begin{aligned}
& \left(\mathbf{r R} s_{1} s_{2} \mid p l m, P L M, S M_{S}\right) \\
& \quad=Y_{l m}(\hat{r}) Y_{L M}(\hat{R}) j_{l}(p r) j_{L}(P R) \chi_{S M_{S}}\left(s_{1} s_{2}\right)
\end{aligned}
$$

describes the spherical free waves for the outgoing particles,

$$
\mathbf{r}=\mathbf{r}_{1}-\mathbf{r}_{2}, \quad \mathbf{R}=\frac{1}{2}\left(\mathbf{r}_{1}+\mathbf{r}_{2}\right)
$$

are the relative and c.m. coordinates, and $l$ and $L$ are the quantum numbers for the relative (l) and c.m. (L) orbital angular momenta. After performing the angular integration in Eq. (2.3) we obtain

$\mathcal{I}(p, P)$

$$
=\sum_{S M_{S} J_{F} M_{F}} \sum_{l m L M}\left|\left\langle p l m, P L M, S M_{S} ; J_{F} M_{F}|V| J_{I} M_{I}\right\rangle\right|^{2},
$$

which goes into

$$
\mathcal{I}(p, P)=\sum_{S l L \lambda J J_{F}}\left|\left\langle p P l L \lambda S J_{,} J_{F} ; J_{I} M_{I}|V| J_{I} M_{I}\right\rangle\right|^{2}
$$

when the angular momentum couplings $\mathbf{I}+\mathbf{L}=\boldsymbol{\lambda}, \boldsymbol{\lambda}+\mathbf{S}=\mathbf{J}$ are carried out. The quantum number $M_{I}$ is superfluous and will be omitted from now on.

The transition potential is written in the Fock space as

$$
\begin{aligned}
V= & \sum_{l L \lambda S J j_{N} j_{\Lambda}}\left\langle p P l L \lambda S J|V| j_{\Lambda} j_{N} J\right\rangle \\
& \times\left(a_{p l(1 / 2)}^{\dagger} a_{P L(1 / 2)}^{\dagger}\right)_{\lambda S J}\left(a_{j_{N}}^{-} a_{j_{\Lambda}}^{-}\right)_{J},
\end{aligned}
$$

where, in the same way as in Eq. (2.1), a transformation to the relative and c.m. momenta is implied. Here $j_{\Lambda}$ and $j_{N}$ are the single-particle shell model states of the decaying particles, and $a_{j m}=(-)^{j+m} a_{j-m}$ [49]. One gets

$$
\begin{aligned}
\mathcal{I}(p, P)= & \hat{J}_{I}^{-2} \sum_{S I L \lambda J J_{F}^{\alpha}} \mid \sum_{j_{N} j_{\Lambda}}\left\langle p P l L \lambda S J|V| j_{\Lambda} j_{N} J\right\rangle \\
& \times\left.\left\langle J_{I}\left\|\left(a_{j_{N}}^{\dagger} a_{j_{\Lambda}}^{\dagger}\right)_{J}\right\| J_{F}^{\alpha}\right\rangle\right|^{2}
\end{aligned}
$$

where the transition amplitudes $\left\langle J_{I}\left\|\left(a_{j_{N}}^{\dagger} a_{j_{\Lambda}}^{\dagger}\right)_{J}\right\| J_{F}\right\rangle$ are reduced with respect to the angular momenta, the label $\alpha$ stands for different final states with the same spin $J_{F}$, and $\hat{J} \equiv \sqrt{2 J+1}$. 
The effective weak hypernuclear interaction is isospin dependent, i.e.,

$$
V\left(\mathbf{r}, s_{\Lambda} s_{N}, t_{\Lambda} t_{N}\right)=\sum_{\tau=0,1} \mathcal{V}_{T}\left(\mathbf{r}, s_{\Lambda} s_{N}\right) \mathcal{T}_{\tau}, \quad \mathcal{T}_{\tau}=\left\{\begin{array}{l}
1, \\
\boldsymbol{\tau}_{\Lambda} \cdot \boldsymbol{\tau}_{N},
\end{array}\right.
$$

and therefore the nuclear matrix elements have to be evaluated in the isospin formalism. This implies Eq. (2.11) goes into

$$
\begin{aligned}
\mathcal{I}_{m_{t_{N}}}(p, P)= & \hat{J}_{I}^{-2} \sum_{S \lambda l L T J J_{F}^{\alpha}} \mid \sum_{j_{N} j_{\Lambda}} \mathcal{M}\left(p P l L \lambda S J T ; j_{\Lambda} j_{N}, m_{t_{N}}\right) \\
& \times\left.\left\langle J_{I}\left\|\left(a_{j_{N} m_{t_{N}}}^{\dagger} a_{j_{\Lambda}}^{\dagger}\right)_{J}\right\| J_{F}^{\alpha}\right\rangle\right|^{2}
\end{aligned}
$$

where

$$
\begin{aligned}
\mathcal{M}\left(p P l L \lambda S J T ; j_{\Lambda} j_{N}, m_{t_{N}}\right) & \\
= & \frac{1}{\sqrt{2}}\left[1-(-)^{l+S+T}\right] \sum_{\tau}\left(p P l L \lambda S J\left|\mathcal{V}_{\tau}\right| j_{\Lambda} j_{N} J\right) \\
& \times\left(T M_{T}=m_{t_{\Lambda}}+m_{t_{N}}\left|\mathcal{T}_{\tau}\right| m_{t_{\Lambda}} m_{t_{N}}\right)
\end{aligned}
$$

is the antisymmetrized nuclear matrix element, and $m_{t_{p}}=\frac{1}{2}$ and $m_{t_{\Lambda}} \equiv m_{t_{n}}=-\frac{1}{2}$. It is assumed, as usual [13], that $\Lambda$ behaves as a $\left|\frac{1}{2},-\frac{1}{2}\right\rangle$ isospin state. In that way, the phenomenological $\Delta T=\frac{1}{2}$ rule is incorporated into the effective interaction. Note that in Eqs. (2.13) and (2.14), $m_{t_{N}}=M_{T}-m_{t_{\Lambda}}$.

To evaluate $\left(p P l L \lambda S J\left|\mathcal{V}_{\tau}\right| j_{\Lambda} j_{N} J\right)$ one has to carry out the $j j-L S$ recoupling and the Moshinsky transformation [50] on the ket $\left.\mid j_{\Lambda} j_{N} J\right)$ to get

$$
\begin{aligned}
& \left(p P l L \lambda S J\left|\mathcal{V}_{\tau}\right| j_{\Lambda} j_{N} J\right) \\
& =\hat{j}_{\Lambda} \hat{j}_{N} \sum_{\lambda^{\prime} S^{\prime} \mathrm{nINL}} \hat{\lambda}^{\prime} \hat{S}^{\prime}\left\{\begin{array}{ccc}
l_{\Lambda} & \frac{1}{2} & j_{\Lambda} \\
l_{N} & \frac{1}{2} & j_{N} \\
\lambda^{\prime} & S^{\prime} & J
\end{array}\right\} \\
& \quad \times\left(\operatorname{nINL} \lambda^{\prime} \mid n_{\Lambda} l_{\Lambda} n_{N} l_{N} \lambda^{\prime}\right) \\
& \quad \times\left(p P l L \lambda S J\left|\mathcal{V}_{\tau}\right| \mathrm{nINL} \lambda^{\prime} S^{\prime} J\right)
\end{aligned}
$$

where $(\cdots \mid \cdots)$ are the Moshinsky brackets [50]. Here I and L stand for the quantum numbers of the relative and c.m. orbital angular momenta in the $\Lambda N$ system. The explicit expressions for the transition potentials are given in the next section, and the formulas that are needed to evaluate the matrix elements $\left(p P l L \lambda S J\left|\mathcal{V}_{\tau}\right| \mathrm{nINL} \lambda^{\prime} S^{\prime} J\right) \quad$ and $\left(T M_{T}\left|\mathcal{I}_{\tau}\right| m_{t_{\Lambda}} m_{t_{N}}\right)$ are summarized in the Appendix.

When the hyperon is assumed to be weakly coupled to the $A-1$ core, which implies that the interaction of $\Lambda$ with core nucleons is disregarded, one has that $\left|J_{I}\right\rangle \equiv\left|\left(J_{C} j_{\Lambda}\right) J_{I}\right\rangle$, where $J_{C}$ is the spin of the core. From

$$
\begin{aligned}
& \left\langle J_{I}\left\|\left(a_{j_{N} m_{t_{N}}}^{\dagger} a_{j_{\Lambda}}^{\dagger}\right)_{J}\right\| J_{F}\right\rangle
\end{aligned}
$$

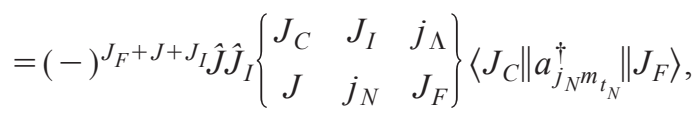

we obtain

$$
\begin{aligned}
\mathcal{I}_{m_{t_{N}}}(p, P)= & \sum_{S I L \lambda J T J_{F}^{\alpha}} \hat{J}^{2} \mid \sum_{j_{N}} \mathcal{M}\left(p P l L \lambda S J T ; j_{\Lambda} j_{N}, m_{t_{N}}\right) \\
& \times\left.\left\{\begin{array}{ccc}
J_{C} & J_{I} & j_{\Lambda} \\
J & j_{N} & J_{F}
\end{array}\right\}\left\langle J_{C}\left\|a_{j_{N} m_{t_{N}}^{\dagger}}^{\dagger}\right\| J_{F}^{\alpha}\right\rangle\right|^{2} \cdot
\end{aligned}
$$

Occasionally it could be convenient to include the isospin coupling as well into $\left\langle J_{C}\left\|a_{j_{N} m_{t_{N}}^{\dagger}}\right\| J_{F}\right\rangle$ and work with the spinisospin reduced parentage coefficients

$\left\langle J_{C} T_{C}\left\|\left|a_{j_{N}(1 / 2)}^{\dagger}\right|\right\| J_{F} T_{F}\right\rangle=\hat{T}_{C} \frac{\left\langle J_{C} T_{C} M_{T_{C}}\left\|a_{j_{N} m_{t_{N}}}^{\dagger}\right\| J_{F} T_{F} M_{T_{F}}\right\rangle}{\left(T_{F} M_{T_{F}} \frac{1}{2} m_{t_{N}} \mid T_{C} M_{T_{C}}\right)}$,

where $T_{C}, M_{T_{C}}$ and $T_{F}, M_{T_{F}}$ are the isospin quantum numbers of the core and residual nuclei, respectively. In this case

$$
\begin{aligned}
\mathcal{I}_{m_{t_{N}}}(p, P)= & \hat{T}_{C}^{-2} \sum_{J_{F}^{\alpha} T_{F} S l L \lambda J T} \hat{J}^{2}\left(T_{F} M_{T_{F}} \frac{1}{2} m_{t_{N}} \mid T_{C} M_{T_{C}}\right)^{2} \\
& \times \mid \sum_{j_{N}} \mathcal{M}\left(p P l L \lambda S J ; j_{\Lambda} j_{N}, m_{t_{N}}\right) \\
& \times\left.\left\{\begin{array}{ccc}
J_{C} & J_{I} & j_{\Lambda} \\
J & j_{N} & J_{F}
\end{array}\right\}\left\langle J_{C} T_{C}\left\|\left|a_{j_{N}(1 / 2)}^{\dagger}\right|\right\| J_{F}^{\alpha} T_{F}\right\rangle\right|^{2} .
\end{aligned}
$$

Thus, knowing the transition potential $V$ and the initial and final nuclear wave functions $\left|J_{I}\right\rangle$ and $\left|J_{F}\right\rangle$ (or $\left|J_{C}\right\rangle$ and $\left|J_{F}\right\rangle$ ), we can evaluate the transition rate (2.4), with the integrations going up to

$$
\Delta_{j_{N} m_{t_{N}}}=M_{\Lambda}-M_{N}+\epsilon_{j_{\Lambda}}+\epsilon_{j_{N} m_{t_{N}}}
$$

where $\epsilon_{j_{\Lambda}}$ and $\epsilon_{j_{N} m_{t_{N}}}$ are the single-particle energies.

\section{EFFECTIVE INTERACTION}

As the reduction of the relativistic one-meson-exchange $t$ matrix, to the nonrelativistic effective potential $V$, is in the literature $[4,6,10-13,24,34,37]$, it will not be repeated here. For the parity-conserving (PC) potential we will just list a 
few results that are indispensable for establishing the notation and for the final discussion. More attention will be given to the parity-violating (PV) potentials. In dealing with them some tricky details appear concerning the passage from momentum space to coordinate space. We first illustrate the procedure for one pseudoscalar meson $(\pi)$ and one vector meson $(\rho)$, and afterwards we generalize the results to all six mesons.

The effective strong $(S)$ and weak $(W)$ Hamiltonians read

$$
\begin{gathered}
\mathcal{H}_{N N \pi}^{S}=i g_{N N \pi} \bar{\psi}_{N} \gamma_{5} \boldsymbol{\pi} \cdot \boldsymbol{\tau} \psi_{N}, \\
\mathcal{H}_{N N \rho}^{S}=\bar{\psi}_{N}\left(g_{N N \rho}^{V} \gamma^{\mu}+i g_{N N \rho}^{T} \frac{\sigma^{\nu \mu} \partial_{\nu}}{2 M}\right) \boldsymbol{\rho}_{\mu} \cdot \boldsymbol{\tau} \psi_{N}, \\
\mathcal{H}_{\Lambda N \pi}^{W}=i G_{F} m_{\pi}^{2} \bar{\psi}_{N}\left(A_{\pi}+B_{\pi} \gamma_{5}\right) \boldsymbol{\tau} \cdot \boldsymbol{\tau} \psi_{\Lambda}\left(\begin{array}{l}
0 \\
1
\end{array}\right),
\end{gathered}
$$

$$
\begin{aligned}
\mathcal{H}_{\Lambda N \rho}^{W}= & G_{F} m_{\pi}^{2} \bar{\psi}_{N}\left(A_{\rho} \gamma^{\mu} \gamma_{5}+B_{\rho}^{V} \gamma^{\mu}+i B_{\rho}^{T} \frac{\sigma^{\nu \mu} \partial_{\nu}}{2 \bar{M}}\right) \\
& \times \boldsymbol{\rho}_{\mu} \cdot \boldsymbol{\tau} \psi_{\Lambda}\left(\begin{array}{l}
0 \\
1
\end{array}\right),
\end{aligned}
$$

where $G_{F} m_{\pi}^{2}$ is the weak coupling constant, $\psi_{N}$ and $\psi_{\Lambda}$ are the baryon fields, $\boldsymbol{\tau}$ and $\boldsymbol{\rho}$ are the meson fields, $\boldsymbol{\tau}$ is the isospin operator, $M$ the nucleon mass, and $\bar{M}$ the average between the nucleon and $\Lambda$ masses. The isospin spurion $\left(\begin{array}{l}0 \\ 1\end{array}\right)$ is included in order to enforce the empirical $\Delta T=\frac{1}{2}$ rule [13].

The corresponding nonrelativistic $t$ matrix in momentum space (with the hyperon $\Lambda$ being always in the first vertex) is

$$
\begin{aligned}
& t_{\pi}(\mathbf{q})=-\boldsymbol{\tau}_{\Lambda} \cdot \boldsymbol{\tau}_{N} \frac{\mathcal{A}_{\pi}\left(\boldsymbol{\sigma}_{N} \cdot \mathbf{q}\right)+\mathcal{B}_{\pi}\left(\boldsymbol{\sigma}_{\Lambda} \cdot \mathbf{q}\right)\left(\boldsymbol{\sigma}_{N} \cdot \mathbf{q}\right)}{m_{\pi}^{2}+\mathbf{q}^{2}} \\
& t_{\rho}(\mathbf{q}, \mathbf{Q})=- \boldsymbol{\tau}_{\Lambda} \cdot \boldsymbol{\tau}_{N} \\
& \times i \mathcal{A}_{\rho}\left(\boldsymbol{\sigma}_{\Lambda} \times \boldsymbol{\sigma}_{N}\right) \cdot \mathbf{q}-2 \mathcal{A}_{\rho}^{\prime} \boldsymbol{\sigma}_{\Lambda} \cdot \mathbf{Q}+\mathcal{B}_{\rho}\left(\boldsymbol{\sigma}_{\Lambda} \times \mathbf{q}\right)\left(\boldsymbol{\sigma}_{N} \times \mathbf{q}\right)-\mathcal{B}_{\rho}^{\prime} \\
& m_{\rho}^{2}+\mathbf{q}^{2}
\end{aligned}
$$

where the coupling constants $\mathcal{A}_{M}, \mathcal{A}_{M}^{\prime}, \mathcal{B}_{M}$, and $\mathcal{B}_{M}^{\prime}$ are defined in Table I and

$$
\mathbf{q}=\mathbf{p}^{\prime}-\mathbf{p}, \quad \mathbf{Q}=\frac{1}{2}\left(\mathbf{p}^{\prime}+\mathbf{p}\right),
$$

with $\mathbf{p}^{\prime}$ and $\mathbf{p}$ being, respectively, the relative momenta for the initial and final states. [We have adopted this labeling to be consistent with Eq. (2.2).] In momentum space the potential reads

$$
\left\langle\mathbf{p}_{1} \mathbf{p}_{2}|V| \mathbf{p}_{1}^{\prime} \mathbf{p}_{2}^{\prime}\right\rangle=-(2 \pi)^{3} \delta\left(\mathbf{p}_{1}^{\prime}+\mathbf{p}_{2}^{\prime}-\mathbf{p}_{1}-\mathbf{p}_{2}\right) t(\mathbf{q}, \mathbf{Q}),
$$

and in order to arrive at coordinate space the Fourier transform is applied:

$$
\begin{aligned}
\left\langle\mathbf{r}_{1} \mathbf{r}_{2}|V| \mathbf{r}_{1}^{\prime} \mathbf{r}_{2}^{\prime}\right\rangle & \\
= & \int \frac{d \mathbf{p}_{1}^{\prime}}{(2 \pi)^{3}} \frac{d \mathbf{p}_{2}^{\prime}}{(2 \pi)^{3}} \frac{d \mathbf{p}_{1}}{(2 \pi)^{3}} \frac{d \mathbf{p}_{2}}{(2 \pi)^{3}}\left\langle\mathbf{p}_{1} \mathbf{p}_{2}|V| \mathbf{p}_{1}^{\prime} \mathbf{p}_{2}^{\prime}\right\rangle \\
& \times \exp \left\{i\left[\mathbf{p}_{1}^{\prime} \cdot \mathbf{r}_{1}^{\prime}+\mathbf{p}_{2}^{\prime} \cdot \mathbf{r}_{2}^{\prime}-\mathbf{p}_{1} \cdot \mathbf{r}_{1}-\mathbf{p}_{2} \cdot \mathbf{r}_{2}\right]\right\}
\end{aligned}
$$

After some trivial integrations and the coordinate transformation

$$
\mathbf{x}=\mathbf{r}-\mathbf{r}^{\prime}, \quad \mathbf{X}=\frac{1}{2}\left(\mathbf{r}^{\prime}+\mathbf{r}\right)
$$

we get

$$
\begin{aligned}
\left\langle\mathbf{r}_{1} \mathbf{r}_{2}|V| \mathbf{r}_{1}^{\prime} \mathbf{r}_{2}^{\prime}\right\rangle= & -\delta\left(\mathbf{R}^{\prime}-\mathbf{R}\right) \int \frac{d \mathbf{Q}}{(2 \pi)^{3}} \frac{d \mathbf{q}}{(2 \pi)^{3}} \\
& \times \exp [i(\mathbf{Q} \cdot \mathbf{x}+\mathbf{q} \cdot \mathbf{X})] t(\mathbf{q}, \mathbf{Q}) .
\end{aligned}
$$

To carry out the integration on $\mathbf{q}$ and $\mathbf{Q}$ we make use of the result

$$
\begin{gathered}
\int \frac{d \mathbf{Q}}{(2 \pi)^{3}} \frac{d \mathbf{q}}{(2 \pi)^{3}} \mathbf{q} \frac{e^{i(\mathbf{Q} \cdot \mathbf{x}+\mathbf{q} \cdot \mathbf{X})}}{m_{M}^{2}+\mathbf{q}^{2}}=-i \delta\left(\mathbf{r}^{\prime}-\mathbf{r}\right) \mathbf{f}_{M}^{(-)}(r), \\
\int \frac{d \mathbf{Q}}{(2 \pi)^{3}} \frac{d \mathbf{q}}{(2 \pi)^{3}} \mathbf{Q} \frac{e^{i(\mathbf{Q} \cdot \mathbf{x}+\mathbf{q} \cdot \mathbf{X})}}{m_{M}^{2}+\mathbf{q}^{2}}=\frac{i}{2} \delta\left(\mathbf{r}^{\prime}-\mathbf{r}\right) \mathbf{f}_{M}^{(+)}(r), \\
\int \frac{d \mathbf{Q}}{(2 \pi)^{3}} \frac{d \mathbf{q}}{(2 \pi)^{3}}\left(\boldsymbol{\sigma}_{1} \cdot \mathbf{q}\right)\left(\boldsymbol{\sigma}_{2} \cdot \mathbf{q}\right) \frac{e^{i(\mathbf{Q} \cdot \mathbf{x}+\mathbf{q} \cdot \mathbf{x})}}{m_{M}^{2}+\mathbf{q}^{2}} \\
=-\delta\left(\mathbf{r}^{\prime}-\mathbf{r}\right)\left[f_{M}^{S}(r)\left(\boldsymbol{\sigma}_{1} \cdot \boldsymbol{\sigma}_{2}\right)+f_{M}^{T}(\mathbf{r}) S_{12}(\hat{r})\right], \\
\frac{d \mathbf{Q}}{(2 \pi)^{3}} \frac{d \mathbf{q}}{(2 \pi)^{3}}\left(\boldsymbol{\sigma}_{1} \times \mathbf{q}\right)\left(\boldsymbol{\sigma}_{2} \times \mathbf{q}\right) \frac{e^{i(\mathbf{Q} \cdot \mathbf{x}+\mathbf{q} \cdot \mathbf{x})}}{m_{M}^{2}+\mathbf{q}^{2}} f \\
=-\delta\left(\mathbf{r}^{\prime}-\mathbf{r}\right)\left[2 f_{M}^{S}(r)\left(\boldsymbol{\sigma}_{1} \cdot \boldsymbol{\sigma}_{2}\right)-f_{M}^{T}(r) S_{12}(\hat{r})\right],
\end{gathered}
$$

where 
TABLE I. Isoscalar $(\tau=0)$ and isovector $(\tau=1)$ coupling constants in units of $G_{F} m_{\pi}^{2}=2.21 \times 10^{-7}$.

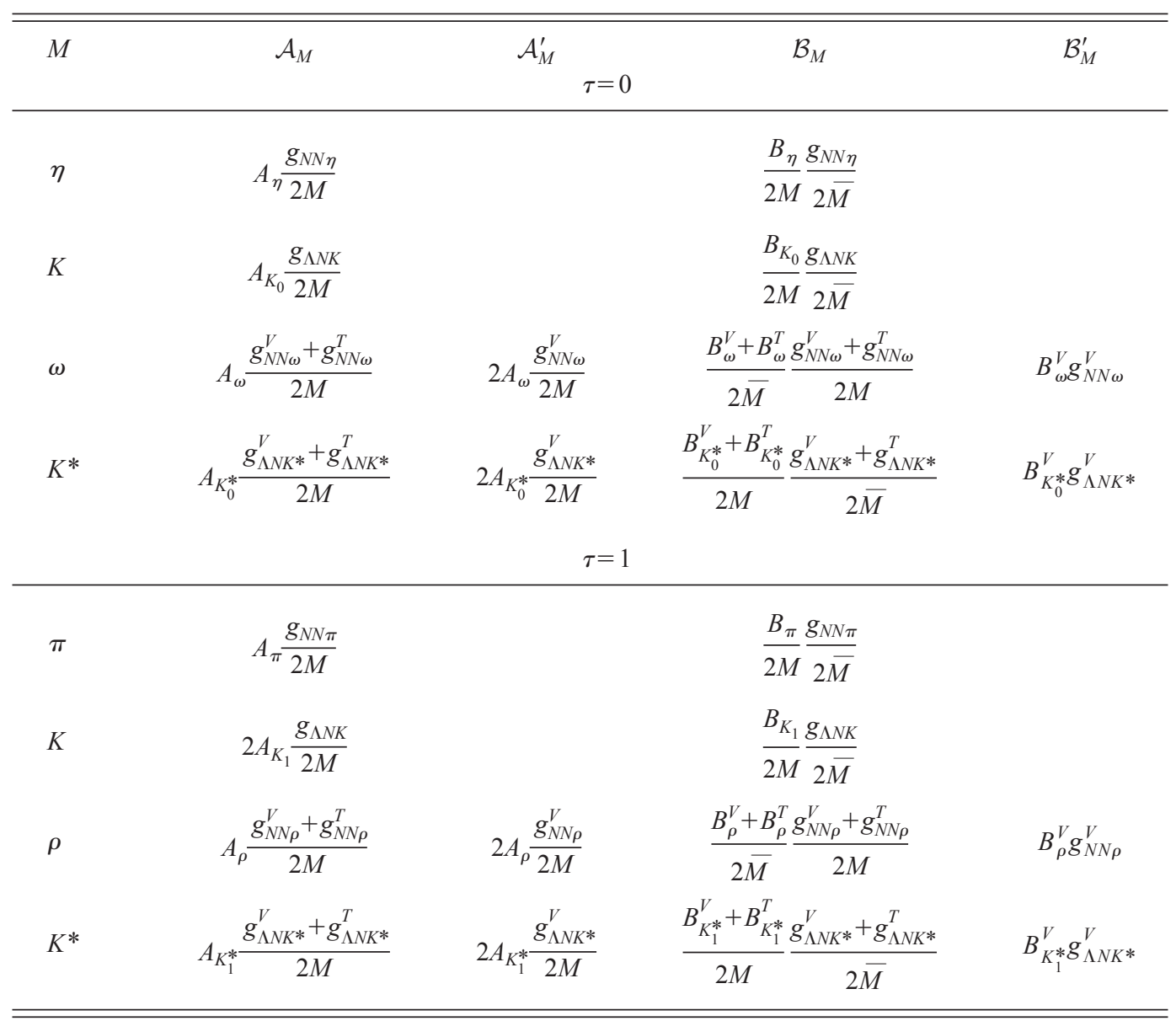

$$
\begin{aligned}
S_{12}(\hat{r}) & =3\left(\boldsymbol{\sigma}_{1} \cdot \hat{r}\right)\left(\boldsymbol{\sigma}_{2} \cdot \hat{r}\right)-\left(\boldsymbol{\sigma}_{1} \cdot \boldsymbol{\sigma}_{2}\right) \\
& =\sqrt{\frac{24 \pi}{5}} Y_{2}(\hat{r}) \cdot\left[\boldsymbol{\sigma}_{1} \times \boldsymbol{\sigma}_{2}\right]_{2},
\end{aligned}
$$

is the tensor operator and the radial dependence is contained in

$$
\begin{gathered}
\mathbf{f}_{M}^{(-)}(r)=\left[\boldsymbol{\nabla}, f_{M}(r)\right]=\boldsymbol{\nabla} f_{M}(r) \equiv \hat{r} \frac{\partial}{\partial r} f_{M}(r)=\hat{r} f_{M}^{\prime}(r), \\
\mathbf{f}_{M}^{(+)}(r)=\left\{\boldsymbol{\nabla}, f_{M}(r)\right\}=\boldsymbol{\nabla} f_{M}(r)+2 f_{M}(r) \boldsymbol{\nabla}, \\
f_{M}^{S}(r)=\frac{1}{3}\left[m_{M}^{2} f_{M}(r)-\delta(\mathbf{r})\right], \\
f_{M}^{T}(r)=\frac{m_{M}^{2}}{3}\left[1+\frac{3}{m_{M} r}+\frac{3}{\left(m_{M} r\right)^{2}}\right] f_{M}(r),
\end{gathered}
$$

with $\boldsymbol{\nabla} \equiv \nabla_{12}=\nabla_{1}=-\nabla_{2}$, and

$$
\begin{gathered}
f_{M}(r)=\frac{e^{-m_{M} r}}{4 \pi r}, \quad r=\left|\mathbf{r}_{1}-\mathbf{r}_{2}\right|, \\
f_{M}^{\prime}(r)=-m_{M}\left(1+\frac{1}{m_{M} r}\right) f_{M}(r) .
\end{gathered}
$$

Thus Eq. (3.7) reads

$$
\left\langle\mathbf{r}_{1} \mathbf{r}_{2}|V| \mathbf{r}_{1}^{\prime} \mathbf{r}_{2}^{\prime}\right\rangle=\delta\left(\mathbf{r}^{\prime}-\mathbf{r}\right) \delta\left(\mathbf{R}^{\prime}-\mathbf{R}\right) V(\mathbf{r}),
$$

where the transition potential for the $\pi+\rho$ model is

$$
\begin{aligned}
V_{\pi+\rho}(\mathbf{r}) & \\
= & \boldsymbol{\tau}_{\Lambda} \cdot \boldsymbol{\tau}_{N}\left\{\left(\boldsymbol{\sigma}_{\Lambda} \cdot \boldsymbol{\sigma}_{N}\right)\left[\mathcal{B}_{\pi} f_{\pi}^{S}(r)+2 \mathcal{B}_{\rho} f_{\rho}^{S}(r)\right]+S_{\Lambda N}(\hat{r})\right. \\
& \times\left[\mathcal{B}_{\pi} f_{\pi}^{T}(r)-\mathcal{B}_{\rho} f_{\rho}^{T}(r)\right]+\mathcal{B}_{\rho}^{\prime} f_{\rho}(r)-i \mathcal{A}_{\pi} \boldsymbol{\sigma}_{N} \cdot \mathbf{f}_{\pi}^{(-)}(r) \\
& \left.-i \mathcal{A}_{\rho}^{\prime} \boldsymbol{\sigma}_{\Lambda} \cdot \mathbf{f}_{\rho}^{(+)}(r)+\mathcal{A}_{\rho}\left(\boldsymbol{\sigma}_{\Lambda} \times \boldsymbol{\sigma}_{N}\right) \cdot \mathbf{f}_{\rho}^{(-)}(r)\right\} .
\end{aligned}
$$

The complete potential can now be cast in the form (2.12), with the isoscalar $(\eta, \omega)$ and isovector $(\pi, \rho)$ mesons giving rise to $\mathcal{V}_{0}$ and $\mathcal{V}_{1}$, respectively, while the strange mesons $\left(K, K^{*}\right)$ contribute to both. We get

$$
\begin{aligned}
\mathcal{V}_{0}^{\mathrm{PV}}\left(\mathbf{r}, s_{1} s_{2}\right)= & -i \boldsymbol{\sigma}_{N} \cdot\left[\mathcal{A}_{\eta} \mathbf{f}_{\eta}^{(-)}(r)-\mathcal{A}_{K_{0}^{*}}^{\prime} \mathbf{f}_{K^{*}}^{(+)}(r)\right] \\
& +i \boldsymbol{\sigma}_{\Lambda} \cdot\left[\mathcal{A}_{K_{0}} \mathbf{f}_{K}^{(-)}(r)-\mathcal{A}_{\omega}^{\prime} \mathbf{f}_{\omega}^{(+)}(r)\right] \\
& +\left(\boldsymbol{\sigma}_{\Lambda} \times \boldsymbol{\sigma}_{N}\right) \cdot\left[\mathcal{A}_{\omega} \mathbf{f}_{\omega}^{(-)}(r)+\mathcal{A}_{K_{0}^{\prime}} \mathbf{f}_{K^{*}}^{(-)}(r)\right],
\end{aligned}
$$




$$
\begin{aligned}
\mathcal{V}_{1}^{\mathrm{PV}}\left(\mathbf{r}, s_{1} s_{2}\right)= & -i \boldsymbol{\sigma}_{N} \cdot\left[\mathcal{A}_{\pi^{\prime}} \mathbf{f}_{\pi}^{(-)}(r)-\mathcal{A}_{K_{1}^{*}}^{\prime} \mathbf{f}_{K^{*}}^{(+)}(r)\right] \\
& +i \boldsymbol{\sigma}_{\Lambda} \cdot\left[\mathcal{A}_{K_{1}} \mathbf{f}_{K}^{(-)}(r)-\mathcal{A}_{\rho}^{\prime} \mathbf{f}_{\rho}^{(+)}(r)\right] \\
& +\left(\boldsymbol{\sigma}_{\Lambda} \times \boldsymbol{\sigma}_{N}\right) \cdot\left[\mathcal{A}_{\rho} \mathbf{f}_{\rho}^{(-)}(r)+\mathcal{A}_{K_{1}^{* *}} \mathbf{f}_{K^{*}}^{(-)}(r)\right],
\end{aligned}
$$

for the PV potential, and

$$
\begin{aligned}
\mathcal{V}_{0}^{\mathrm{PC}}\left(\mathbf{r}, s_{1} s_{2}\right)= & \left(\boldsymbol{\sigma}_{\Lambda} \cdot \boldsymbol{\sigma}_{N}\right)\left[\mathcal{B}_{\eta} f_{\eta}^{S}(r)+\mathcal{B}_{K_{0}} f_{K}^{S}(r)+2 \mathcal{B}_{\omega} f_{\omega}^{S}(r)\right. \\
& \left.+2 \mathcal{B}_{K_{0}^{*}} f_{K^{*}}^{S}(r)\right]+S_{\Lambda N}(\hat{r})\left[\mathcal{B}_{\eta} f_{\eta}^{T}(r)\right. \\
& \left.+\mathcal{B}_{K_{0}} f_{K}^{T}(r)-\mathcal{B}_{\omega} f_{\omega}^{T}(r)-\mathcal{B}_{K_{0}^{*}} f_{K^{*}}^{T}(r)\right] \\
& +\mathcal{B}_{\omega}^{\prime} f_{\omega}(r)+\mathcal{B}_{K_{0}^{*}}^{\prime} f_{K^{*}}(r), \\
\mathcal{V}_{1}^{\mathrm{PC}}\left(\mathbf{r}, s_{1} s_{2}\right)= & \left(\boldsymbol{\sigma}_{\Lambda} \cdot \boldsymbol{\sigma}_{N}\right)\left[\mathcal{B}_{\pi} f_{\pi}^{S}(r)+\mathcal{B}_{K_{1}} f_{K}^{S}(r)+2 \mathcal{B}_{\rho} f_{\rho}^{S}(r)\right. \\
& \left.+2 \mathcal{B}_{K_{1}^{*}} f_{K^{*}}^{S}(r)\right]+S_{\Lambda N}(\hat{r})\left[\mathcal{B}_{\pi} f_{\pi}^{T}(r)\right. \\
& \left.+\mathcal{B}_{K_{1}} f_{K}^{T}(r)-\mathcal{B}_{\rho} f_{\rho}^{T}(r)-\mathcal{B}_{K_{1}^{*}} f_{K^{*}}^{T}(r)\right] \\
& +\mathcal{B}_{\rho}^{\prime} f_{\rho}(r)+\mathcal{B}_{K_{1}^{\prime}}^{\prime} f_{K^{*}}(r),
\end{aligned}
$$

for the PC potential. The overall coupling constants $\mathcal{A}_{M}$, $\mathcal{A}_{M}^{\prime}, \mathcal{B}_{M}$, and $\mathcal{B}_{M}^{\prime}$ are listed in Table I, with the weak couplings for kaons defined as

$$
\begin{gathered}
A_{K_{0}}=\frac{C_{K}^{\mathrm{PV}}}{2}+D_{K}^{\mathrm{PV}}, \quad A_{K_{1}}=\frac{C_{K}^{\mathrm{PV}}}{2}, \\
B_{K_{0}}=\frac{C_{K}^{\mathrm{PC}}}{2}+D_{K}^{\mathrm{PC}}, \quad B_{K_{1}}=\frac{C_{K}^{\mathrm{PC}}}{2}, \\
A_{K_{0}^{*}}=\frac{C_{K^{*}}^{\mathrm{PV}}}{2}+D_{K^{*}}^{\mathrm{PV}}, \quad A_{K_{1}^{*}}=\frac{C_{K^{*}}^{\mathrm{PV}}}{2}, \\
B_{K_{0}^{*}}^{V}=\frac{C_{K^{*}}^{\mathrm{PC}, V}}{2}+D_{K^{*}, V}^{\mathrm{PC},}, \quad B_{K_{1}^{*}}^{V}=\frac{C_{K^{*}}^{\mathrm{PC}, V}}{2}, \\
B_{K_{0}^{*}}^{T}=\frac{C_{K^{*}}^{\mathrm{PC}, T}}{2}+D_{K^{*}}^{\mathrm{PC}, T}, \quad B_{K_{1}^{*}}^{T}=\frac{C_{K^{*}}^{\mathrm{PC}, T}}{2} .
\end{gathered}
$$

The $C^{\prime}$ 's and $D$ 's are given in Ref. [13]. The operators that have been habitually omitted in $\mathcal{V}^{\mathrm{PV}}\left(\mathbf{r}, s_{1} s_{2}\right)$ are those that are proportional to $\mathcal{A}_{M}^{\prime}$.

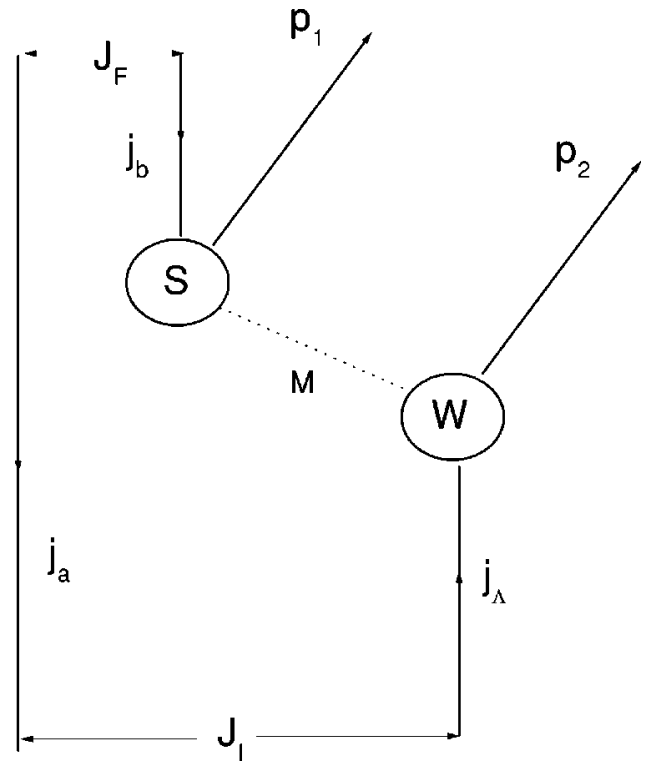

FIG. 1. Diagramatic representation of the hypernuclear NM weak decay, from the one-particle-one-hole (1p1h) state $\left|j_{\Lambda} j_{a}^{-1} ; J_{I}\right\rangle$ to the $2 \mathrm{~h}$ state $\left|j_{a}^{-1} j_{b}^{-1} J_{F}\right\rangle$, while two nucleons with momenta $\mathbf{p}_{1}$ and $\mathbf{p}_{2}$ are emitted into the continuum. $S$ and $W$ are the strong and weak vertices, respectively, and $M$ is a nonstrange meson.

\section{NUCLEAR MODELS AND MULTIPOLE EXPANSION}

\section{A. Extreme particle-hole model}

The simplest nuclear shell model is the EPHM, in which the hypernucleus ${ }_{\Lambda}^{A} Z$ is described as a $\Lambda$ hyperon in the single-particle state $\left|j_{\Lambda}\right\rangle$ and a hole state $\left|j_{a}^{-1}\right\rangle$ relative to the ${ }^{A} Z$ core, while the residual ${ }^{A-2} Z$ and ${ }^{A-2}(Z-1)$ nuclei are represented by the two-hole states $\left|j_{a}^{-1} j_{b}^{-1}\right\rangle$ with respect to the same core. As illustrated in Fig. $1,\left|J_{I}\right\rangle \rightarrow\left|j_{\Lambda} j_{a}^{-1} ; J_{I}\right\rangle$, $\left|J_{F}\right\rangle \rightarrow\left|j_{a}^{-1} j_{b}^{-1} ; J_{F}\right\rangle$, and $\left|J_{C}\right\rangle \rightarrow\left|j_{a}^{-1}\right\rangle$. The parentage coefficients Eq. (2.17) read

$$
\left\langle J_{C}\left\|a_{j_{b} m_{t_{b}}}^{\dagger}\right\| J_{F}\right\rangle=(-)^{J_{F}+j_{a}+j_{b}} \sqrt{1+\delta_{a b}} \hat{J}_{F} .
$$

In particular, for ${ }_{\Lambda}^{12} C$ the initial state is

$$
\left(a_{j \Lambda}^{\dagger} \overline{a_{j_{a}}}\right)_{J_{I}}|0\rangle \equiv\left|j_{\Lambda} j_{a}^{-1} ; J_{I}\right\rangle=\left|1 s_{1 / 2} \Lambda, 1 p_{3 / 2} n^{-1} ; 1\right\rangle,
$$

and the final states are

$$
\begin{aligned}
\left(\overline{a_{j}} \bar{a}_{j_{b}}\right)_{J_{F}}|0\rangle & \equiv\left|j_{a}^{-1} j_{b}^{-1} ; J_{F}\right\rangle \\
& =\left\{\begin{array}{l}
\left|\left(1 p_{3 / 2} n^{-1}\right)^{2} ; 0,2\right\rangle,\left|1 p_{3 / 2} n^{-1} 1 s_{1 / 2} n^{-1} ; 1,2\right\rangle, \\
\left|\left(1 p_{3 / 2} n^{-1} 1 p_{3 / 2} p^{-1}\right) ; 0,1,2,3\right\rangle,\left|1 p_{3 / 2} n^{-1} 1 s_{1 / 2} p^{-1} ; 1,2\right\rangle
\end{array}\right.
\end{aligned}
$$


TABLE II. Geometrical factors $\hat{j}_{b}^{2} F_{m_{t_{b}}}^{j_{b}}\left(j_{a}\right)$.

\begin{tabular}{lcccc}
\hline \hline$j_{a}$ & $j_{b}$ & $J$ & Neutrons & Protons \\
\hline $1 p_{3 / 2}$ & $1 s_{1 / 2}$ & 0 & 1 & 1 \\
& & 1 & 3 & 3 \\
$1 p_{1 / 2}$ & $1 s_{1 / 2}$ & 0 & 1 & 1 \\
& & 1 & 3 & 3 \\
\hline $1 p_{3 / 2}$ & $1 p_{3 / 2}$ & 1 & 7 & 6 \\
$1 p_{1 / 2}$ & $1 p_{3 / 2}$ & 1 & 5 & 10 \\
& & 2 & 10 & 6 \\
$1 p_{3 / 2}$ & $1 p_{1 / 2}$ & 0 & 1 & 10 \\
$1 p_{1 / 2}$ & $1 p_{1 / 2}$ & 0 & 3 & 3 \\
& & 1 & 0 & 3 \\
\hline \hline
\end{tabular}

for $\Lambda n \rightarrow n n$ and $\Lambda p \rightarrow n p$, respectively. Here $|0\rangle$ is the ${ }^{12} \mathrm{C}$ particle vacuum. As there is only one hole state for each parity, the parentage coefficients with different $j_{b}=j_{N}$ do not interfere among themselves. After summing up the final states the integrand (2.17) can be cast in the form

$$
\begin{aligned}
\mathcal{I}_{m_{t_{b}}} & (p, P) \\
& =\sum_{j_{b} J} F_{m_{t_{b}} J}^{j_{b}}\left(p_{3 / 2}\right) \sum_{S \lambda l L T} \mathcal{M}^{2}\left(p P l L \lambda S J T ; j_{\Lambda} j_{b}, m_{t_{b}}\right),
\end{aligned}
$$

where

$$
\begin{aligned}
F_{m_{t_{b}} J}^{j_{b}}= & \hat{J}^{2} \sum_{J_{F}=\mid j_{j_{a}-j_{b} \mid}^{j_{a}+j_{b}}\left[1+(-)^{J_{F}} \delta_{j_{a} j_{b}} \delta_{m_{t_{b}} m_{t_{a}}}\right]} \\
& \times \hat{J}_{F}^{2}\left\{\begin{array}{ccc}
j_{a} & j_{b} & J_{F} \\
J & J_{I} & j_{\Lambda}
\end{array}\right\}^{2}
\end{aligned}
$$

are geometrical factors which come from the Pauli principle. Their explicit values for the $1 s_{1 / 2}, 1 p_{3 / 2}$, and $1 p_{1 / 2}$ are listed in Table II.

\section{B. Beyond the extreme particle-hole model}

The EPHM can be straightforwardly improved by going to the quasiparticle representation. In fact, for all even-mass hypernuclei the initial and final states can be expressed as

$$
\begin{aligned}
\left|J_{I}\right\rangle & =\sum_{j_{\Lambda} j_{a}} C_{j_{\Lambda} j_{a}}\left(a_{j_{\Lambda}}^{\dagger} b_{j_{a}}^{\dagger}\right)_{J_{I}}|\mathrm{BCS}\rangle, \\
\left|J_{F}^{\alpha}\right\rangle & =\sum_{j_{a} j_{b}} C_{j_{a} j_{b} J_{F}}^{\alpha}\left(b_{j_{a}}^{\dagger} b_{j_{b}}^{\dagger}\right)_{J_{F}}|\mathrm{BCS}\rangle,
\end{aligned}
$$

where $b_{j}^{\dagger}=u_{j} a_{j}^{\dagger}-v_{j} a_{j}^{-}$is the quasiparticle creation operator [48] and $|\mathrm{BCS}\rangle$ is the proton-neutron BCS vacuum. Note that $j_{a}$ is always a neutron state, while $j_{b}$ can be both a neutron and proton orbital. Note that because of the lack of hyperon-hole states, the backward-going RPA contributions do not appear and one has to work within the QTDA. From Eq. (2.13) we get

$$
\begin{aligned}
\mathcal{I}_{m_{t_{b}}}(p, P)= & \sum_{l L \lambda S J J_{F}^{\alpha} T} \hat{J}^{2} \hat{J}_{F}^{2} \mid \sum_{j_{\Lambda} j_{a} j_{b}}(-)^{j_{a}+j_{b}} \sqrt{1+\delta_{a b}} \\
& \times C_{j_{a} j_{b} J_{F}}^{\alpha} C_{j_{a} j_{\Lambda}} v_{j_{b}} \mathcal{M}\left(p P l L \lambda S J T ; j_{\Lambda} j_{b}, m_{t_{b}}\right) \\
& \times\left.\left\{\begin{array}{ccc}
j_{a} & J_{I} & j_{\Lambda} \\
J & j_{b} & J_{F}
\end{array}\right\}\right|^{2}
\end{aligned}
$$

The residual interaction in the final nuclei redistributes the transition rates among the states with the same spin and parity. But as the NM decay is an inclusive process-i.e., the partial transition rates are summed up coherently over all final states - such a rearrangement plays only a very minor role on the total rates. (The same happens, for instance, in the neutrino-nucleus reactions and in $\mu$-meson capture [51].) Therefore, it is justifiable to approximate the final wave functions by their unperturbed forms, i.e., $\left|J_{F}^{\alpha}\right\rangle$ $\equiv\left(b_{j_{a}}^{\dagger} b_{j_{b}}^{\dagger}\right)_{J_{F}}|\mathrm{BCS}\rangle$ and $C_{j_{a} j_{b} J_{F}}^{\alpha} \equiv \delta_{\alpha, j_{a} j_{b}}$. If, in addition, one assumes that the hyperon is always in the lowest $1 s_{1 / 2}$ state, the last equation takes the form of Eq. (4.4), i.e.,

$$
\begin{aligned}
\mathcal{I}_{m_{t_{b}}}(p, P)= & \sum_{j_{a} j_{b} J} F_{m_{t_{b}}}^{j_{b}}\left(j_{a}\right) C_{j_{a} j_{\Lambda}}^{2} v_{j_{b}}^{2} \\
& \times \sum_{S \lambda l L T} \mathcal{M}^{2}\left(p P l L \lambda S J T ; j_{\Lambda} j_{b}, m_{t_{b}}\right) .
\end{aligned}
$$

Only the orbitals $1 s_{1 / 2}, 1 p_{3 / 2}$, and $1 p_{1 / 2}$ will be used. In this case, as seen from Table II, $F_{p J}^{j_{b}}\left(p_{1 / 2}\right)=F_{p J}^{j_{b}}\left(p_{3 / 2}\right)$, which implies that in the case of protons the summation on $j_{a}$ can be performed analytically. Thus, as $\Sigma_{j_{a}} C_{j_{a} j_{\Lambda}}^{2}=1$, one finds out that $\Gamma_{p}$ does not depend at all on the initial wave function. From the same table one also finds out that $F_{n J}^{j_{b}}\left(j_{a}\right)$ $=F_{p J}^{j_{b}}\left(j_{a}\right)$, except when $j_{b}=j_{a}$. So one can expect as well only a weak dependence of $\Gamma_{n}$ on $\left|J_{I}\right\rangle$. This fact is verified numerically later on.

In summary, we end up with a very simple result for the transition rates:

$$
\Gamma_{m_{t_{b}}}=\sum_{j_{b} J} v_{j_{b}}^{2} F_{m_{t_{b}} J}^{j_{b}}\left(j_{a}\right) \mathcal{R}_{m_{t_{b}} J}^{j_{b}}
$$

where

$$
\begin{aligned}
\mathcal{R}_{m_{t_{b}} J}^{j_{b}}= & \frac{16 M_{N}^{3} \int_{0}^{\Delta_{j_{b}}} d \epsilon \sqrt{\epsilon\left(\Delta_{j_{b}}-\epsilon\right)}}{\pi} \\
& \times \sum_{\operatorname{SlL\lambda } T} \mathcal{M}^{2}\left(p \operatorname{PlL} \lambda S J T ; j_{\Lambda} j_{b}, m_{t_{b}}\right) .
\end{aligned}
$$

Clearly, the EPHM is contained in Eq. (4.9) with the occupation numbers $v_{j_{b}}$ equal to 1 for the occupied states and to 0 for the empty states. 


\section{Multipole expansion}

The EPHM is particularly suitable for performing the multipole expansion of the integrands $\mathcal{I}_{m_{t_{b}}}$. Thus we carry out both the Racah algebra in Eq. (2.15) and the summations indicated in Eq. (4.4), keeping in mind that the allowed quantum numbers $\{\mathrm{IL}\}$ are $\{00\}$ for the $s_{1 / 2}$ state and $\{01\}$ and $\{10\}$ for the $p_{3 / 2}$ state. To simplify the results we take advantage of the relations

$$
\begin{gathered}
(P 0 \mid 10)=\left(\frac{\pi}{2}\right)^{1 / 4} b^{3 / 2} e^{-(P b)^{2} / 4} \\
(P 1 \mid 11)=\frac{1}{\sqrt{3}}\left(\frac{\pi}{2}\right)^{1 / 4} b^{5 / 2} P e^{-(P b)^{2} / 4}
\end{gathered}
$$

for the radial integrals $(P L \mid \mathrm{NL})$ defined in Eq. (A2) and introduce the ratio

$$
R=\frac{(b P)^{2}}{3} \equiv\left[\frac{(P 1 \mid 11)}{(P 0 \mid 10)}\right]^{2},
$$

which allows us to work only with the $L=0$ overlap $(P 0 \mid 10)$. Thus, from now on, the label $L$ will be disregarded, and to identify the $s_{1 / 2}$ and $p_{3 / 2}$ pieces of the I=0 strength we will use the ratio $R$, which appears only in the last term of Eq. (4.4). The results of the multipole expansion for both PC and PV potentials are displayed below.

\section{Parity-conserving contributions}

The matrix elements of the PC operators $f_{M}(r), f_{M}^{S}(r)$ $\times\left(\boldsymbol{\sigma}_{\Lambda} \cdot \boldsymbol{\sigma}_{N}\right)$, and $f_{M}^{T}(r) S_{\Lambda N}(\hat{r})$, given by Eq. (A1), can be expressed by means of the radial matrix elements (A2) and (A3) or, more precisely, through the moments

$$
\mathrm{C}_{M}^{\mathrm{l}}(p, P)=\mathcal{B}_{M}^{\prime}\left(p|| f_{M} \mid 1 \mathrm{I}\right)(P 0 \mid 10),
$$

$$
\begin{gathered}
\mathrm{S}_{M}^{\prime}(p, P)=\mathcal{B}_{M}\left(p|| f_{M}^{S} \mid 1 \mathrm{I}\right)(P 0 \mid 10) \begin{cases}1 & \text { for } \pi, \eta, K, \\
2 & \text { for } \rho, \omega, K^{*},\end{cases} \\
\mathrm{T}_{M}^{l l}(p, P)=\mathcal{B}_{M}\left(p l\left|f_{M}^{T}\right| 1 \mathrm{I}\right)(P 0 \mid 10) \times \begin{cases}1 & \text { for } \pi, \eta, K, \\
-1 & \text { for } \rho, \omega, K^{*} .\end{cases}
\end{gathered}
$$

Introducing the notation

$$
\begin{array}{ll}
\tau=0 & \tau=1 \\
\overline{\mathrm{C}}_{0}=\mathrm{C}_{\omega}+\mathrm{C}_{K_{0}}, & \overline{\mathrm{C}_{1}=\mathrm{C}_{\rho}+\mathrm{C}_{K_{1}^{*}},} \\
\mathrm{~S}_{0}=\mathrm{S}_{\eta}+\mathrm{S}_{\omega}+\mathrm{S}_{K_{0}}+\mathrm{S}_{K_{0}^{*},}, \mathrm{~S}_{1}=\mathrm{S}_{\pi}+\mathrm{S}_{\rho}+\mathrm{S}_{K_{1}}+\mathrm{S}_{K_{1}^{*},}, \\
\mathrm{~T}_{0}=\mathrm{T}_{\eta}+\mathrm{T}_{\omega}+\mathrm{T}_{K_{0}}+\mathrm{T}_{K_{0}^{*}}, & \mathrm{~T}_{1}=\mathrm{T}_{\pi}+\mathrm{T}_{\rho}+\mathrm{T}_{K_{1}}+\mathrm{T}_{K_{1}^{*},}
\end{array}
$$

for the isoscalar $(\tau=0)$ and the isovector $(\tau=1)$ matrix elements, one gets

$$
\begin{aligned}
\mathcal{I}_{p}= & 2(1+R)\left[3\left(\mathrm{~S}_{0}^{0}\right)^{2}+9\left(\mathrm{~S}_{1}^{0}\right)^{2}+\left(\mathrm{C}_{0}^{0}\right)^{2}+7\left(\mathrm{C}_{1}^{0}\right)^{2}+6\left(3 \mathrm{~T}_{1}^{20}\right.\right. \\
& \left.\left.-\mathrm{T}_{0}^{20}\right)^{2}-4 \mathrm{C}_{0}^{0} \mathrm{C}_{1}^{0}+12 \mathrm{C}_{1}^{0} \mathrm{~S}_{1}^{0}-6 \mathrm{C}_{0}^{0} \mathrm{~S}_{1}^{0}-6 \mathrm{C}_{1}^{0} \mathrm{~S}_{0}^{0}\right]+6\left(\mathrm{~S}_{0}^{1}\right)^{2} \\
& +42\left(\mathrm{~S}_{1}^{1}\right)^{2}-24 \mathrm{~S}_{0}^{1} \mathrm{~S}_{1}^{1}+2\left(\mathrm{C}_{0}^{1}\right)^{2}+6\left(\mathrm{C}_{1}^{1}\right)^{2}-24 \mathrm{C}_{1}^{1} \mathrm{~S}_{1}^{1} \\
& +12 \mathrm{C}_{1}^{1} \mathrm{~S}_{0}^{1}+12 \mathrm{C}_{0}^{1} \mathrm{~S}_{1}^{1}+\frac{6}{5}\left(\mathrm{~T}_{0}^{11}+\mathrm{T}_{1}^{11}\right)^{2}+\frac{54}{5}\left(\mathrm{~T}_{0}^{31}+\mathrm{T}_{1}^{31}\right)^{2}
\end{aligned}
$$

for the decay $\Lambda p \rightarrow n p$, and

$$
\begin{aligned}
\mathcal{I}_{n}= & \left(1+\frac{7 R}{3}\right)\left(3 \mathrm{~S}_{0}^{0}+3 \mathrm{~S}_{1}^{0}-\mathrm{C}_{0}^{0}-\mathrm{C}_{1}^{0}\right)^{2}+\frac{11}{6}\left(\mathrm{~S}_{0}^{1}+\mathrm{C}_{0}^{1}+\mathrm{S}_{1}^{1}\right. \\
& \left.+\mathrm{C}_{1}^{1}\right)^{2}+\frac{38}{15}\left(\mathrm{~T}_{0}^{11}+\mathrm{T}_{1}^{11}\right)^{2}+\frac{54}{5}\left(\mathrm{~T}_{0}^{31}+\mathrm{T}_{1}^{31}\right)^{2}
\end{aligned}
$$

for the decay $\Lambda n \rightarrow n n$.

\section{Parity-violating contributions}

The PV matrix elements (A5) are reduced to the nuclear moments

$$
\begin{aligned}
& \mathrm{P}_{M}^{l l}(p, P)=\mathcal{A}_{M}\left(p l\left|f_{M}^{(-)}\right| 1 \mathrm{I}\right)(P 0 \mid 10), \\
& \mathrm{Q}_{M}^{l l}(p, P)=\mathcal{A}_{M}^{\prime}\left(p l\left|f_{M}^{(+)}\right| 1 \mathrm{I}\right)(P 0 \mid 10),
\end{aligned}
$$

where the radial integrals $\left(p l\left|f_{M}^{( \pm)}\right| 1 \mathrm{I}\right)$ are defined in Eq. (A8). Using the notation

$$
\begin{aligned}
& \tilde{\mathrm{P}}_{\eta}=\mathrm{P}_{\eta}-\mathrm{Q}_{K_{0}^{*}}, \quad \tilde{\mathrm{P}}_{K_{0}}=\mathrm{P}_{K_{0}}-\mathrm{Q}_{\omega}, \quad \tilde{\mathrm{P}}_{K_{0}^{*}}=\mathrm{P}_{K_{0}^{*}}+\mathrm{P}_{\omega}, \\
& \tilde{\mathrm{P}}_{\pi}=\mathrm{P}_{\pi}-\mathrm{Q}_{K_{1}^{*}}, \quad \tilde{\mathrm{P}}_{K_{1}}=\mathrm{P}_{K_{1}}-\mathrm{Q}_{\rho}, \quad \tilde{\mathrm{P}}_{K_{1}^{*}}=\mathrm{P}_{K_{1}^{*}}+\mathrm{P}_{\rho},
\end{aligned}
$$

we obtain 


$$
\begin{aligned}
\mathcal{I}_{p}= & 2(1+R)\left[3\left(\tilde{\mathrm{P}}_{\pi}^{10}\right)^{2}+\left(\tilde{\mathrm{P}}_{\eta}^{10}\right)^{2}+3\left(\tilde{\mathrm{P}}_{K_{1}}^{10}\right)^{2}+\left(\tilde{\mathrm{P}}_{K_{0}}^{10}\right)^{2}+10\left(\tilde{\mathrm{P}}_{K_{1}^{*}}^{10}\right)^{2}+2\left(\tilde{\mathrm{P}}_{K_{0}^{*}}^{10}\right)^{2}-2 \tilde{\mathrm{P}}_{\eta}^{10} \tilde{\mathrm{P}}_{K_{1}}^{10}+2 \tilde{\mathrm{P}}_{\pi}^{10}\left(2 \tilde{\mathrm{P}}_{K_{1}}^{10}-\tilde{\mathrm{P}}_{K_{0}}^{10}+4 \tilde{\mathrm{P}}_{K_{1}^{*}}^{10}-2 \tilde{\mathrm{P}}_{K_{0}^{*}}^{10}\right)\right. \\
& \left.+4 \tilde{\mathrm{P}}_{K_{1}}^{10}\left(2 \tilde{\mathrm{P}}_{K_{1}^{*}}^{10}-\tilde{\mathrm{P}}_{K_{0}^{*}}^{10}\right)-4 \tilde{\mathrm{P}}_{K_{1}^{*}}^{10} \tilde{\mathrm{P}}_{K_{0}^{*}}^{10}\right]+14\left(\tilde{\mathrm{P}}_{\pi}^{21}\right)^{2}+2\left(\tilde{\mathrm{P}}_{\eta}^{21}\right)^{2}+8\left(\tilde{\mathrm{P}}_{K_{1}}^{21}\right)^{2}+\frac{4}{3}\left(\tilde{\mathrm{P}}_{K_{0}}^{21}\right)^{2}+14\left(\tilde{\mathrm{P}}_{K_{1}^{*}}^{21}\right)^{2}+\frac{10}{3}\left(\tilde{\mathrm{P}}_{K_{0}^{*}}^{21}\right)^{2}+4 \tilde{\mathrm{P}}_{\eta}^{21} \tilde{\mathrm{P}}_{K_{1}}^{21} \\
& -4 \tilde{\mathrm{P}}_{\pi}^{21}\left(2 \tilde{\mathrm{P}}_{K_{0}^{*}}^{21}+2 \tilde{\mathrm{P}}_{K_{1}}^{21}-\tilde{\mathrm{P}}_{K_{0}}^{21}+4 \tilde{\mathrm{P}}_{K_{1}^{*}}^{21}-2 \tilde{\mathrm{P}}_{K_{0}^{*}}^{21}\right)+4 \tilde{\mathrm{P}}_{K_{1}}^{21}\left(\tilde{\mathrm{P}}_{\eta}^{21}-\tilde{\mathrm{P}}_{K_{1}^{*}}^{21}+\tilde{\mathrm{P}}_{K_{0}^{*}}^{21}\right)+4 \tilde{\mathrm{P}}_{K_{1}^{*}}^{21}\left(2 \tilde{\mathrm{P}}_{\eta}^{21}-\tilde{\mathrm{P}}_{K_{0}^{*}}^{21}\right)+\frac{4}{3} \tilde{\mathrm{P}}_{K_{0}^{*}}^{21} \tilde{\mathrm{P}}_{K_{0}}^{21}+\frac{2}{3}\left(\tilde{\mathrm{P}}_{K_{0}}^{01}\right)^{2} \\
& +6\left(\tilde{\mathrm{P}}_{K_{1}}^{01}\right)^{2}+\frac{2}{3}\left(\tilde{\mathrm{P}}_{K_{0}^{*}}^{01}\right)^{2}+6\left(\tilde{\mathrm{P}}_{K_{1}^{*}}^{01}\right)^{2}-4 \tilde{\mathrm{P}}_{K_{0}}^{01} \tilde{\mathrm{P}}_{K_{1}}^{01}-\frac{4}{3} \tilde{\mathrm{P}}_{K_{0}^{*}}^{01}\left(\tilde{\mathrm{P}}_{K_{0}}^{01}-3 \tilde{\mathrm{P}}_{K_{1}}^{01}+3 \tilde{\mathrm{P}}_{K_{1}^{*}}^{01}\right)+4 \tilde{\mathrm{P}}_{K_{1}^{*}}^{01}\left(\tilde{\mathrm{P}}_{K_{0}}^{01}-3 \tilde{\mathrm{P}}_{K_{1}}^{01}\right),
\end{aligned}
$$

for the $\Lambda p \rightarrow n p$ decay, and

$$
\begin{aligned}
\mathcal{I}_{n}= & \left(3+\frac{43 R}{18}\right)\left[\left(\tilde{\mathrm{P}}_{\pi}^{10}+\tilde{\mathrm{P}}_{\eta}^{10}\right)^{2}+\left(\tilde{\mathrm{P}}_{K_{0}}^{10}+\tilde{\mathrm{P}}_{K_{1}}^{10}\right)^{2}\right]+\left(4+\frac{14 R}{3}\right)\left(\tilde{\mathrm{P}}_{K_{0}^{*}}^{10}+\tilde{\mathrm{P}}_{K_{1}^{*}}^{10}\right)^{2}-\left(2+\frac{R}{9}\right)\left(\tilde{\mathrm{P}}_{\pi}^{10}+\tilde{\mathrm{P}}_{\eta}^{10}\right)\left(\tilde{\mathrm{P}}_{K_{0}}^{10}+\tilde{\mathrm{P}}_{K_{1}}^{10}\right)-\left(4+\frac{14 R}{3}\right)\left(\tilde{\mathrm{P}}_{K_{0}^{*}}^{10}\right. \\
& \left.+\tilde{\mathrm{P}}_{K_{1}^{*}}^{10}\right)\left(\tilde{\mathrm{P}}_{\pi}^{10}+\tilde{\mathrm{P}}_{\eta}^{10}+\tilde{\mathrm{P}}_{K_{0}}^{10}+\tilde{\mathrm{P}}_{K_{1}}^{10}\right)+\frac{1}{2}\left(\tilde{\mathrm{P}}_{\pi}^{21}+\tilde{\mathrm{P}}_{\eta}^{21}+\tilde{\mathrm{P}}_{K_{0}}^{21}+\tilde{\mathrm{P}}_{K_{1}}^{21}\right)^{2}+2\left(\tilde{\mathrm{P}}_{K_{0}^{*}}^{21}+\tilde{\mathrm{P}}_{K_{1}^{*}}^{21}\right)\left(\tilde{\mathrm{P}}_{K_{0}^{*}}^{21}+\tilde{\mathrm{P}}_{K_{1}^{*}}^{21}+\tilde{\mathrm{P}}_{\pi}^{21}+\tilde{\mathrm{P}}_{\eta}^{21}+\tilde{\mathrm{P}}_{K_{0}}^{21}+\tilde{\mathrm{P}}_{K_{1}}^{21}\right), \quad(4.20)
\end{aligned}
$$

for the $\Lambda n \rightarrow n n$ decay.

\section{NUMERICAL RESULTS AND DISCUSSION}

The numerical values of the parameters, defined in Table I and necessary to specify the transition potential, are summarized in Table III. For the sake of comparison all cutoffs appearing in Eqs. (5.1), as well as all coupling constants, were taken from Ref. [13], where, in turn, the strong couplings have been taken from Refs. $[28,29]$ and the weak ones from Ref. [12]. The energy difference $\Delta_{j_{N} m_{t_{N}}}$ in Eq. (2.20) is evaluated from the experimental single-nucleon and hyperon energies, quoted in Ref. [8].

The finite nucleon size (FNS) effects at the interaction vertices are gauged by the monopole form factor $F_{M}^{(\mathrm{FNS})}$ $\times\left(\mathbf{q}^{2}\right)=\left(\Lambda_{M}^{2}-m_{M}^{2}\right) /\left(\Lambda_{M}^{2}+\mathbf{q}^{2}\right)$, which implies that the propagators in Eqs. (3.10) and (3.11) must be replaced by

$$
f_{M}(r) \rightarrow \overline{f_{M}}(r)=f_{M}(r)-f_{\Lambda_{M}}(r)-\frac{r\left(\Lambda_{M}^{2}-m_{M}^{2}\right)}{2 \Lambda_{M}} f_{\Lambda_{M}}(r),
$$

$$
\begin{aligned}
f_{M}^{S}(r) \rightarrow & \overline{f_{M}^{S}}(r)=f_{M}^{S}(r)-f_{\Lambda_{M}}^{S}(r) \\
& -\frac{1}{6}\left(\Lambda_{M}^{2}-m_{M}^{2}\right)\left(\Lambda_{M} r-2\right) f_{\Lambda_{M}}(r), \\
f_{M}^{T}(r) \rightarrow & \overline{f_{M}^{T}}(r)=f_{M}^{T}(r)-f_{\Lambda_{M}}^{T}(r) \\
& -\frac{1}{6}\left(\Lambda_{M}^{2}-m_{M}^{2}\right)\left(\Lambda_{M} r+1\right) f_{\Lambda_{M}}(r), \\
f_{M}^{\prime}(r) \rightarrow \overline{f_{M}^{\prime}}(r)= & f_{M}^{\prime}(r)-f_{\Lambda_{M}^{\prime}}^{\prime}(r)+\frac{r\left(\Lambda_{M}^{2}-m_{M}^{2}\right)}{2} f_{\Lambda_{M}}(r),
\end{aligned}
$$

\begin{tabular}{|c|c|c|c|c|c|c|}
\hline \multicolumn{4}{|c|}{$\tau=0$} & $\mathcal{A}_{M}^{\prime} / m_{M}$ & $\mathcal{B}_{M}$ & $\mathcal{B}_{M}^{\prime} / m_{M}^{2}$ \\
\hline$\eta$ & 548.6 & 1.3 & 0.247 & & -0.525 & \\
\hline$K$ & 495.8 & 1.2 & -0.828 & & 0.228 & \\
\hline$\omega$ & 783.4 & 1.5 & -0.274 & -0.420 & -0.923 & -1.395 \\
\hline$K^{*}$ & 892.4 & 2.2 & 0.376 & 0.237 & 0.632 & 1.016 \\
\hline \multicolumn{7}{|c|}{$\tau=1$} \\
\hline$\pi$ & 140.0 & 1.3 & 1.175 & & -0.546 & \\
\hline$K$ & 495.8 & 1.2 & -0.127 & & 0.764 & \\
\hline$\rho$ & 775.0 & 1.4 & 0.273 & 0.105 & -0.907 & -0.407 \\
\hline$K^{*}$ & 892.4 & 2.2 & 0.514 & 0.324 & 1.072 & 0.274 \\
\hline
\end{tabular}

where $f_{\Lambda_{M}}(r)$ has the same structure as $f_{M}(r)$ but with $m_{M} \rightarrow \Lambda_{M}$.

The initial and final short-range correlations (SRCs) are taken into account, respectively, via the correlation functions [13]

TABLE III. Parameters used in the calculations: masses (in MeV), cutoffs (in $\mathrm{GeV}$ ), and the isoscalar $(\tau=0)$ and isovector $(\tau=1)$ coupling constants (in units of $10^{-11} \mathrm{MeV}^{-2}$ ). 
TABLE IV. Parity-conserving (PC) and parity-violating (PV) nonmesonic decay rates for ${ }_{\Lambda}^{12} C$, in units of $\Gamma^{0}=2.50 \times 10^{-6} \mathrm{eV}$. All coupling constants and the cutoff parameters are from Table III and $b=1.51 \mathrm{fm}$. All calculations were done within the EPHM, except for a few results which were evaluated in the QTDA and are shown parenthetically.

\begin{tabular}{lcccc}
\hline \hline \multicolumn{1}{c}{ Mesons } & $\Gamma_{n}^{\mathrm{PC}}$ & $\Gamma_{n}^{\mathrm{PV}}$ & $\Gamma_{p}^{\mathrm{PC}}$ & $\Gamma_{p}^{\mathrm{PV}}$ \\
\hline$\pi$ & 0.009 & 0.151 & 0.734 & 0.383 \\
& $(0.016)$ & $(0.153)$ & $(0.732)$ & $(0.373)$ \\
$\eta$ & 0.003 & 0.004 & 0.006 & 0.003 \\
$K$ & 0.008 & 0.069 & 0.097 & 0.043 \\
$\rho$ & 0.005 & 0.003 & 0.109 & 0.008 \\
$\omega$ & 0.004 & 0.007 & 0.066 & 0.004 \\
$K^{*}$ & 0.025 & 0.034 & 0.056 & 0.028 \\
\hline$\pi+\eta$ & 0.013 & 0.204 & 0.630 & 0.383 \\
$\pi+K$ & 0.013 & 0.258 & 0.325 & 0.512 \\
$\pi+\rho$ & 0.009 & 0.133 & 0.583 & 0.461 \\
$\pi+\omega$ & 0.015 & 0.176 & 0.902 & 0.406 \\
$\pi+K^{*}$ & 0.044 & 0.075 & 1.020 & 0.455 \\
$\pi+\eta+K$ & 0.008 & 0.318 & 0.259 & 0.505 \\
& $(0.011)$ & $(0.330)$ & $(0.258)$ & $(0.516)$ \\
$\pi+\eta+K+K^{*}$ & 0.052 & 0.268 & 0.486 & 0.602 \\
All mesons & 0.037 & 0.240 & 0.347 & 0.714 \\
& $(0.039)$ & $(0.250)$ & $(0.346)$ & $(0.702)$ \\
\hline \hline
\end{tabular}

$$
\begin{gathered}
g_{i}(r)=\left(1-e^{-r^{2} / \alpha^{2}}\right)^{2}+\beta r^{2} e^{-r^{2} / \gamma^{2}}, \\
g_{f}(r)=1-j_{0}\left(q_{c} r\right),
\end{gathered}
$$

with $\alpha=0.5 \mathrm{fm}, \quad \beta=0.25 \mathrm{fm}^{-2}, \quad \gamma=1.28 \mathrm{fm}, \quad$ and $q_{c}$ $=3.93 \mathrm{fm}^{-1}$.

It is a general belief nowadays that, in any realistic evaluation of the hypernuclear NM decay, the FNSs and SRCs have to be included simultaneously. Therefore, in the present paper we will discuss only the numerical results, in which both of these renormalization effects are considered. Under these circumstances and because of the relative smallness of pion mass, the transition is dominated by the OPE [13].

The major part of the numerical calculations were done in the EPHM where the only free parameter is the harmonic oscillator length $b$. The most commonly used estimate is $b$ $=A^{1 / 6} \mathrm{fm}[49,48]$, which corresponds to the oscillator energy $\hbar \omega=41 A^{-1 / 3} \mathrm{MeV}$ and gives $b=1.51 \mathrm{fm}$. For light nuclei it is sometimes preferred to employ $\hbar \omega=45 A^{-1 / 3}$ $-25 A^{-2 / 3} \mathrm{MeV}$, which yields $b=1.70 \mathrm{fm}$. Moreover, a $\Lambda$ particle in a hypernucleus is typically less bound than the corresponding nucleon and hence $b_{\Lambda}$ could be larger than $b_{N}$. For instance, in Ref. [13] $b=\left(b_{\Lambda}+b_{N}\right) / 2=1.75 \mathrm{fm}$ was used, which comes from $b_{N}=1.64 \mathrm{fm}$ and $b_{\Lambda}=1.87 \mathrm{fm}$. As there is no deep motivation for preferring one particular value of $b$, the numerical results will be exhibited for both $b=1.51$ and $1.75 \mathrm{fm}$.

First, a few illustrative results, obtained in the EPHM [Eq. (4.4)] and the simplified version of the QTDA [Eq. (4.8)], are displayed in the Table IV. The hyperon-nucleon interaction in the latter approach was taken to be a simple $\delta$ force, which has been recently used with success as the nucleon-nucleon interaction to explain the weak decay processes in ${ }^{12} \mathrm{C}[51]$. The resulting pairing BCS factors were $v_{s_{1 / 2}}=0.9868, v_{p_{3 / 2}}$ $=0.8978$ and $v_{p_{1 / 2}}=0.6439$. Although we have expected to obtain small differences between the EPHM and QTDA, it came as a surprise that they turned out to be so tiny. Thus, henceforth, only the first one will be used.

Next, we combine results from Table IV with the multipole expansion done in the previous section to find out the roles played by different mesons. Note that formulas (4.19) and (4.20) depend on the ratio (4.12), and it was found numerically that the approximation

$$
R=1
$$

reproduces fairly well the exact calculations. This estimate helped us to formulate the following comments.

PC potential. The dominant contributions to $\Gamma_{p}$ and $\Gamma_{n}$ come from the $\mathrm{I}=0$ matrix elements, while the $\mathrm{I}=1$ wave contributes relatively little: $\cong 2 \%$ to $\Gamma_{p}$ and $\cong 10 \%$ to $\Gamma_{n}$. On the other hand, for the parametrization displayed in Table III, one finds that (1) the $\omega$ and $K^{*}$ mesons mainly cancel out in $\mathrm{C}_{0}^{0}$, as the $\rho$ and $K^{*}$ mesons do in $\mathrm{C}_{1}^{0}$, and (2) the matrix elements $S_{0}^{0}$ and $S_{1}^{0}$ are small in comparison with $\left(3 T_{1}^{20}\right.$ $-\mathrm{T}_{0}^{20}$ ), which makes $\Gamma_{p}$ large in relation to $\Gamma_{n}$. Thus, using the estimate (5.3), one ends up with the following approximate result for the PC contributions:

$$
\mathcal{I}_{p}+\mathcal{I}_{n} \cong \mathcal{I}_{p} \cong 2 \mathcal{I}_{p}^{s_{1 / 2}} \cong 24\left(3 \mathrm{~T}_{1}^{20}-\mathrm{T}_{0}^{20}\right)^{2}
$$

From Table III and Eq. (4.14) one can also see that (i) the $\omega$ and $K^{*}$ mesons contribute coherently with the pion, while the remaining three mesons contribute out of phase, and (ii) the different vector meson contributions have the tendency to cancel among themselves. As shown in Table IV, the overall effect is a reduction of the pion transition rate by approximately a factor of 2 .

$P V$ potential. As in the $\mathrm{PC}$ case, the dominant $\mathrm{PV}$ transition strengths come from the $\mathrm{I}=0$ wave, through the $\tilde{\mathrm{P}}_{M}^{10}$ moments. The $\mathrm{I}=1$ wave from the $p_{3 / 2}$ state gives rise to $l$ $=0$ and $l=2$ outgoing channels. The first one can always be neglected, while the second one contributes with $15 \%$ to $\Gamma_{p}$ and with $2 \%$ to $\Gamma_{n}$, when only the $\pi$ meson is considered. After including all mesons these percentages drop to $6 \%$ and $1 \%$, respectively. Also here the partial $s_{1 / 2}$ and $p_{3 / 2}$ contributions are approximately equal for all mesons in the protoninduced channel and notably different in the neutron-induced channel.

From Table IV it is easily found that the most important PV contributions arise from the $\mathrm{P}_{\pi}^{10}$ moment and from its interference with the $\mathrm{P}_{\eta}^{10}, \mathrm{P}_{K}^{10}$, and $\mathrm{P}_{K^{*}}^{10}$ moments. Thus, retaining only the most relevant terms in Eqs. (4.19) and (4.20), the following rough estimates are obtained: 


$$
\begin{aligned}
\mathcal{I}_{p} \cong & 2 \mathcal{I}_{p}^{s}{ }^{1 / 2} \cong 4\left[3\left(\mathrm{P}_{\pi}^{10}\right)^{2}+3\left(\mathrm{P}_{K_{1}}^{10}\right)^{2}+\left(\mathrm{P}_{K_{0}}^{10}\right)^{2}\right] \\
& +8 \mathrm{P}_{\pi}^{10}\left(2 \mathrm{P}_{K_{1}}^{10}-\mathrm{P}_{K_{0}}^{10}+4 \mathrm{P}_{K_{1}^{*}}^{10}-2 \mathrm{P}_{K_{0}^{*}}^{10}-3 \mathrm{Q}_{K_{1}^{*}}^{10}\right)
\end{aligned}
$$

and

$$
\begin{aligned}
\mathcal{I}_{n} \cong & \frac{97}{18}\left[\left(\mathrm{P}_{\pi}^{10}\right)^{2}+\left(\mathrm{P}_{K_{1}}^{10}+\mathrm{P}_{K_{0}}^{10}\right)^{2}\right]-\frac{19}{9} \mathrm{P}_{\pi}^{10}\left(\mathrm{P}_{K_{1}}^{10}+\mathrm{P}_{K_{0}}^{10}\right) \\
& +\frac{97}{9} \mathrm{P}_{\pi}^{10}\left(\mathrm{P}_{\eta}^{10}-\mathrm{Q}_{K_{1}^{*}}^{10}-\mathrm{Q}_{K_{0}^{*}}^{10}\right)-\frac{78}{9} \mathrm{P}_{\pi}^{10}\left(\mathrm{P}_{K_{1}^{*}}^{10}+\mathrm{P}_{K_{0}^{*}}^{10}\right) .
\end{aligned}
$$

These relations are notably more complicated than Eq. (5.4). Nevertheless, it can be concluded that (1) the $\eta$ meson is only significant for $\Gamma_{n}$ and (2) the $K$ and $K^{*}$ mesons increase both transition rates, but in a different way.

Before proceeding it is worth saying a few words on the "new" nuclear moments $\mathrm{Q}_{M}^{10}$ and compare them with the well-known moments $\mathrm{P}_{M}^{10}$. As seen from Eqs. (4.17) and (A8)-(A10), they basically differ in the radial dependence. Specifically, we discuss the radial matrix element

$$
\overline{\left(p, 1\left|f_{K^{*}}^{(+)}\right| 10\right)}=\overline{\left(p, 1\left|f_{K^{*}}^{L}\right| 10\right)}+\overline{\left(p, 1\left|f_{K^{*}}^{R}\right| 10\right)},
$$

which appears in $\mathrm{Q}_{K^{*}}^{10}$, together with the usual matrix element

$$
\overline{\left(p, 1\left|f_{K^{*}}^{(-)}\right| 10\right)}=\overline{\left(p, 1\left|f_{K^{*}}^{L}\right| 10\right)}-\overline{\left(p, 1\left|f_{K^{*}}^{R}\right| 10\right)},
$$

which is contained in $\mathrm{P}_{K^{*}}^{10}$. The overbar indicates that both the FNSs and SRCs are included, as explained in the Appendix.

As can be seen from Fig. 2, the matrix elements of $f_{K^{*}}^{L}$ and $f_{K^{*}}^{R}$ have opposite signs, and as a consequence, the matrix element of $f_{K^{*}}^{(-)}$is larger in magnitude than that of $f_{K^{*}}^{(+)}$. A rough approximation for the mean values is

$$
\left|\left\langle\overline{\left(p 1\left|f_{K^{*}}^{(+)}\right| 10\right)}\right\rangle\right| \cong \frac{1}{2}\left|\left\langle\overline{\left(p 1\left|f_{K^{*}}^{(-)}\right| 10\right)}\right\rangle\right| .
$$

As $\mathcal{A}_{K^{*}} \gtrsim \mathcal{A}_{K^{*}}^{\prime}$ (see Table III), we end up with the estimate

$$
\left|\left\langle Q_{K^{*}}^{10}\right\rangle\right| \cong 0.3\left|\left\langle P_{K^{*}}^{10}\right\rangle\right| \text {. }
$$

Thus Eqs. (5.5) and (5.6) show that the $K^{*}$ meson mainly contributes through the moments $\mathrm{P}_{K^{*}}^{10}$, augmenting the magnitude of $\Gamma_{p}^{\mathrm{PV}}$ and diminishing that of $\Gamma_{n}^{\mathrm{PV}}$. The matrix elements $Q_{K^{*}}^{10}$, in contrast, reduce both transition rates.

Furthermore, Eq. (4.18) indicates that each vector moment $\mathrm{Q}_{\rho, \omega, K^{*}}^{10}$ is accompanied by a pseudoscalar moment $\mathrm{P}_{\pi, \eta, K}^{10}$. Both integrals $\left(p 1\left|f_{M}^{( \pm)}\right| 10\right)$ are negative for all mesons. Then, using the values of the coupling constants $\mathcal{A}_{\pi, \eta, K}$ and $\mathcal{A}_{\rho, \omega, K^{*}}^{\prime}$ listed in Table III, it can be inferred that $\mathrm{Q}_{\rho, \omega, K^{*}}^{10}$ and $\mathrm{P}_{\pi, \eta, K}^{10}$ moments mostly add incoherently.

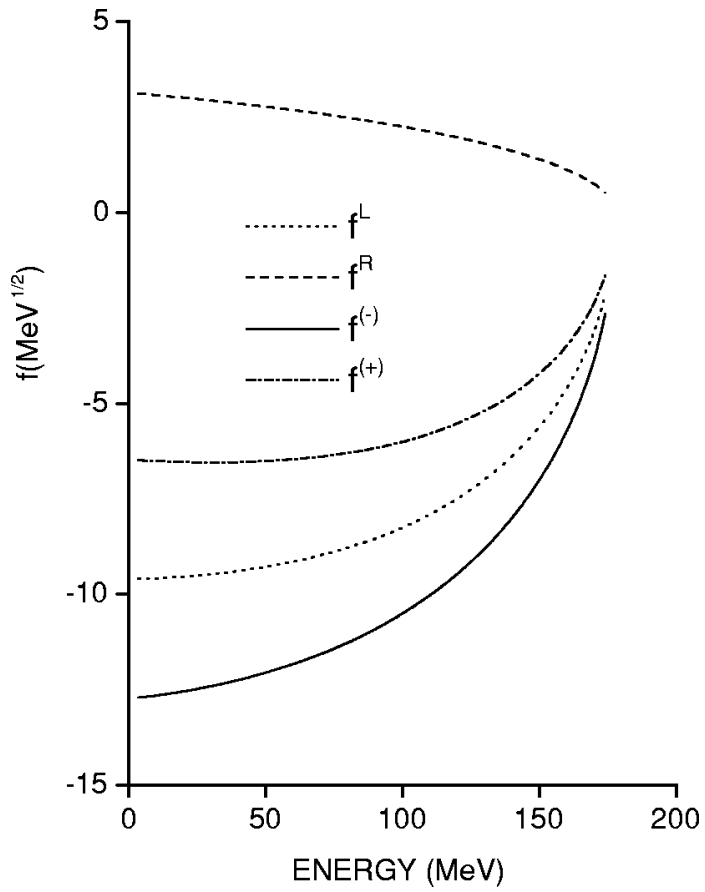

FIG. 2. Matrix elements of the radial operators $f_{K^{*}}^{L}, f_{K^{*}}^{R}, f_{K^{*}}^{(+)}$, and $f_{K^{*}}^{(-)}$, as a function of the energy.

The experimental results for the total transition rate $\Gamma_{\mathrm{NM}}$, the proton partial width $\Gamma_{p}$, and the ratio $\Gamma_{n / p}$ in ${ }_{\Lambda}^{12} C$ are displayed in Table V. In the same table the theoretical estimates are also shown, grouped as follows.

(1) Calculation A. All the parametrization is taken from Table III, and the following cases are shown and commented on:

(i) $(\pi)$ : The simple OPE model accounts for $\Gamma_{\mathrm{NM}}$, but it badly fails regarding $\Gamma_{p}$ and $\Gamma_{n / p}$.

(ii) (PS): When $\eta$ and $K$ mesons are included, the total transition rate is only slightly modified, while $\Gamma_{p}$ and $\Gamma_{n / p}$ change significantly, coming somewhat closer to the measured values.

(iii) $\left(\mathrm{PS}+K^{*}\right)$ : The incorporation of the $K^{*}$ meson increases $\Gamma_{\mathrm{NM}}$ and $\Gamma_{p}$, decreases $\Gamma_{n / p}$, and in this way worsens the agreement with the data.

(iv) $(\mathrm{PS}+V)$ : The results are not drastically modified when all vector mesons are built in.

(v) $(\mathrm{PS}+V(\mathrm{P}))$ : All six mesons are included, but only the $\mathrm{PV}$ moments $\mathrm{P}_{M}$ are considered. The importance of the new moments $\mathrm{Q}_{M}$ is evident from the comparison with the previous case.

The main conclusion is that it is not possible to reproduce simultaneously the data for all three observables $\Gamma_{\mathrm{NM}}, \Gamma_{p}$, and $\Gamma_{n / p}$, when the BBM coupling are constrained by the $\mathrm{SU}(3)$ and $\mathrm{SU}(6)_{w}$ symmetries.

(2) Calculation $B$. We discuss now what happens when the just-mentioned constraints are relaxed, and the FNS and SRC parametrizations, as well as the pion couplings, are kept unchanged. That is, the transition potential is considered to be given by a series of Yukawa-like potentials with different 
TABLE V. Parity-conserving (PC) and parity-violating (PV) nonmesonic decay rates for ${ }_{\Lambda}^{12} C$, in units of $\Gamma^{0}=2.50 \times 10^{-6} \mathrm{eV}$. The data are taken from Refs. [39-43], and large experimental errors are due to the low efficiencies and large backgrounds in neutron detection. The calculations were performed for both $b$ $=1.51$ and $1.75 \mathrm{fm}$, the latter being given parenthetically. In calculation $A$ all parameters are from Table III, and PS and V stand, respectively, for the pseudoscalar $(\pi+\eta+K)$ and the vector $(\rho$ $\left.+\omega+K^{*}\right)$ mesons, while the label $(\mathrm{P})$ indicates that only the moments $\mathrm{P}_{M}$ are considered [see Eq. (4.17)]. In calculation $B$ the coupling constants listed in Table III are modified as $\mathcal{A}_{\eta} \rightarrow 3 \mathcal{A}_{\eta}$, $\mathcal{A}_{K_{1}} \rightarrow 5 \mathcal{A}_{K_{1}}, \mathcal{B}_{\eta} \rightarrow 3 \mathcal{B}_{\eta}$, and $\mathcal{B}_{K} \rightarrow 2 \mathcal{B}_{K}$, and the signs of all vector meson potentials are inverted.

\begin{tabular}{cccc}
\hline \hline & $\Gamma_{\mathrm{NM}}=\Gamma_{n}+\Gamma_{p}$ & $\Gamma_{p}$ & $\Gamma_{n / p}=\Gamma_{n} / \Gamma_{p}$ \\
\hline $\begin{array}{c}\text { Measurements } \\
\text { Ref. [39] }\end{array}$ & & \\
Ref. [39] & & & $0.70 \pm 0.3$ \\
Ref. [40] & $1.14 \pm 0.2$ & & $0.52 \pm 0.16$ \\
Ref. [41] & $0.89 \pm 0.15 \pm 0.03$ & $0.31_{-0.11}^{+0.18}$ & $1.83_{-0.81}^{+1.12}$ \\
Ref. [42] & $1.14 \pm 0.08$ & & \\
Ref. [43] & & & $1.17_{-0.08-0.18}^{+0.32}$ \\
\hline Calculation $A$ & & & \\
$\pi$ & $1.277(1.006)$ & $1.116(0.885)$ & $0.143(0.137)$ \\
PS & $1.100(0.851)$ & $0.774(0.601)$ & $0.420(0.416)$ \\
PS+ $K^{*}$ & $1.408(1.091)$ & $1.088(0.846)$ & $0.294(0.290)$ \\
PS+ V & $1.338(1.038)$ & $1.061(0.825)$ & $0.261(0.259)$ \\
PS+ V(P) & $1.539(1.190)$ & $1.196(0.927)$ & $0.287(0.284)$ \\
\hline Calculation $B$ & & & \\
PS' & $1.145(0.874)$ & $0.555(0.419)$ & $1.064(1.089)$ \\
PS' $-K^{*}$ & $1.273(0.971)$ & $0.540(0.407)$ & $1.355(1.384)$ \\
PS' - V & $1.297(0.989)$ & $0.542(0.408)$ & $1.394(1.423)$ \\
\hline \hline
\end{tabular}

spin and isospin dependences. The simple increase of the $K$ coupling does not solve the problem by itself. For instance, for $\mathcal{A}_{K} \rightarrow 2 \mathcal{A}_{K}$ and $\mathcal{B}_{K} \rightarrow 2 \mathcal{B}_{K}$, the contribution of all three pseudoscalar mesons is (when $b=1.51 \mathrm{fm}$ ): $\Gamma_{\mathrm{NM}}$ $=1.404 \Gamma^{0}, \Gamma_{p}=0.815 \Gamma^{0}$, and $\Gamma_{n / p}=0.723$, and when the vector mesons are added one gets $\Gamma_{\mathrm{NM}}=1.714 \Gamma^{0}, \Gamma_{p}$ $=1.130 \Gamma^{0}$, and $\Gamma_{n / p}=0.518$. Namely, $\Gamma_{\mathrm{NM}}$ turns out to be too large. But from the previous discussion, in relation to Eqs. (5.4), (5.5), and (5.6), we have learned that it could be possible to reproduce at the same time the data for all three observables by (i) making the total tensor interaction in $\Gamma_{p}^{\mathrm{PC}}$ small and simultaneously (ii) decreasing $\Gamma_{p}^{\mathrm{PV}}$ and increasing $\Gamma_{n}^{\mathrm{PV}}$, without modifying $\Gamma_{\mathrm{NM}}$ too much. The first goal can be accomplished, for instance, through the modifications $\mathcal{B}_{\eta}$ $\rightarrow 3 \mathcal{B}_{\eta}$ and $\mathcal{B}_{K} \rightarrow 2 \mathcal{B}_{K}$ and the second one with $\mathcal{A}_{\eta} \rightarrow 3 \mathcal{A}_{\eta}$ and $\mathcal{A}_{K_{1}} \rightarrow 5 \mathcal{A}_{K_{1}}$. The following cases are illustrated in Table $\mathrm{V}$ :

(i) $\left(\mathrm{PS}^{\prime}\right)$ : Only the pseudoscalar mesons are included with the above changes in $\eta$ and $K$ meson couplings.

(ii) $\left(\mathrm{PS}^{\prime}-K^{*}\right)$ : The $K^{*}$ meson potential is incorporated, but with the inverted sign.

(iii) $\left(\mathrm{PS}^{\prime}-V\right)$ : All vector meson potentials are included with the inverted signs.
No best fit to data has been attempted. Yet it is clear that there are many other set of parameters that reproduce reasonably well the data. We wish to stress as well that, when the vector mesons are considered, the correct values of $\Gamma_{n / p}$ are obtained only by overturning the signs of the vector meson potentials.

\section{SUMMARY AND CONCLUSIONS}

A novel shell model formalism for the nonmesonic weak decay of the hypernuclei has been developed. It involves a partial wave expansion of the emitted nucleon waves and preserves naturally the antisymmetrization between the escaping particles and the residual core. The general expression (2.13) is valid for any nuclear model and it shows that the NM transition rates should depend, in principle, on both (i) the weak transition potential, through the elementary transition amplitudes $\mathcal{M}\left(p P l L \lambda S J T ; j_{\Lambda} j_{N}, m_{t_{N}}\right)$, and (ii) the nuclear structure, through the two-particle $N \Lambda$ parentage coefficients $\left\langle J_{I}\left\|\left(a_{j_{N} t_{t_{N}}}^{\dagger} a_{j_{\Lambda}}^{\dagger}\right)_{J}\right\| J_{F}\right\rangle$. The explicit evaluation of the matrix elements $\mathcal{M}$ is illustrated as well.

Two nuclear models for even-mass hypernuclei-namely, the EPHM and QTDA - were worked out in detail, and Eqs. (4.4) and (4.7) were derived. The last one explicitly depends on the initial and final wave functions. But because of (i) the inclusive nature of the nonmesonic decay and (ii) the peculiar properties of the coefficients $F_{m_{t_{b}} J^{j}}^{j_{b}}\left(j_{a}\right)$, this dependence is totally washed out for all practical purposes. In this way we have arrived at a very simple result for the transition rates, given by Eq. (4.9), which except for the BCS pairing factors $v_{j_{b}}^{2}$ agrees with the EPHM result. Thus it can be stated that the two-particle correlations in the initial and final states are only of minor importance if of any. With some additional effort higher-order nuclear structure effects such as the four quasiparticle excitations, collective vibrations, rotations, etc., can also be incorporated. Yet it is hard to imagine a scenario where the latter could be relevant at the same time that the former are not. Therefore, we conclude that the nuclear structure manifests basically through the factor $F_{m_{t_{b}} J^{j}}^{j_{b}}\left(j_{a}\right)$, which is engendered by the Pauli principle; $j_{a}$ stands for the hyperon partner in the initial state, and $j_{b}$ runs over all proton and neutron occupied states in the initial nucleus. It is amazing to notice that Eq. (4.9) is valid for any even-mass system, which can be so light as ${ }_{\Lambda}^{4} \mathrm{H}$ and ${ }_{\Lambda}^{4} \mathrm{He}$ are or so heavy as ${ }_{\Lambda}^{208} \mathrm{~Pb}$ is. (A quite similar result is also obtained for the odd-mass hypernuclei, and this issue will be discussed elsewhere.) One should also add that the last equation contains the same physics as Eq. (5) in Ref. [13] or Eq. (30) in Ref. [27], with the advantage that we do not have to deal with spectroscopic factors. Of course, neither the initial and final wave functions are needed.

Attention has been given to the nonrelativistic approximation, used to derive the weak effective hypernuclear onemeson-exchange potentials (3.14) and (3.15). Errors and misprints that appear in some recent papers $[13,19,24]$ have been corrected, and additional parity-violating vector meson operators $\boldsymbol{\sigma}_{N} \cdot \mathbf{f}^{(+)}(r)$ and $\boldsymbol{\sigma}_{\Lambda} \cdot \mathbf{f}^{(+)}(r)$, usually neglected, 
have been considered as well. The matrix elements of these new terms were fully discussed, and it was found that they are quite important quantitatively and therefore should not be omitted.

With the OMEM parametrization from the literature [13] and keeping the treatment of the FSIs at the simple Jastrowlike level $\left[g(r)=1-j_{0}\left(q_{c} r\right)\right]$, we reproduced satisfactorily the data for the total transition rate $\left(\Gamma_{\mathrm{NM}}^{\mathrm{th}} \cong \Gamma^{0}\right)$, but the $n / p$ ratio $\left(\Gamma_{n / p}^{\text {th }} \lesssim 0.42\right)$ and the proton partial width ( $\Gamma_{p}^{\text {th }}$ $\gtrsim 0.60 \Gamma^{0}$ ) are not well accounted for. More elaborate treatments of the FSIs increase sensibly the $n / p$ ratio, but they are unable to solve the puzzle $[13,24]$, especially after the last experimental result for this observable [43]. We have found that the new vector meson operators are not of much help in this regard either.

Finally, bearing in mind the phenomenological nature of the OMEM, we have also tried to reproduce all three data simultaneously by varying the coupling strengths in a significant way. As the only guide, the simple formulas (5.6), (5.7), and (5.8) were used, which come out from the multipole expansion done within the EPHM. Such an attempt was successful, and we get $0.87 \lesssim \Gamma_{\mathrm{NM}}^{\text {th }} / \Gamma^{0} \lesssim 1.30,1.06 \lesssim \Gamma_{n / p}^{\text {th }}$ $\lesssim 1.42$, and $0.41 \leqq \Gamma_{p}^{\text {th }} / \Gamma^{0} \leqq 0.55$. We are conscious that changing a coupling by up to a factor of 5 , with the sole justification of accounting for the data, is a rather a desperate way out of the $\Gamma_{n / p}$ puzzle. No profound physical significance is attached to the "new" parameters, and it even can be said that such a procedure is not physical. However, after having acquired full control of the nuclear structure involved in the process and after having convinced ourselves that the nuclear structure correlations cannot play a crucial role, we firmly believe that the currently used OMEM should be radically changed. Either its parametrization has to be modified or additional degrees of freedom have to be incorporated, such as the correlated $2 \pi$ from Ref. [27] or the four-baryon point interaction from Ref. [26], avoiding clearly double counting. In fact, it would be very nice to see the outcomes of such studies.

\section{ACKNOWLEDGMENTS}

The authors acknowledge the support of ANPCyT (Argentina) under Grant No. BID 1201/OC-AR (PICT 0304296), of Fundación Antorchas (Argentina) under Grant No. 13740/01-111, and of Croatian Ministry of Science and Technology under Grant No. 119222. D.T. thanks the Abdus Salam ICTP Visiting Scholar program for two travel fares. F.K. and C.B. are supported by CONICET Argentina. One of us (F.K.) would like to thank A. P. Galeão for very helpful and illuminating discussions and for a careful and critical reading of the manuscript.

\section{APPENDIX: NUCLEAR MATRIX ELEMENTS}

Here we evaluate the transition matrix elements that appear in Eq. (2.15) for the potentials $\mathcal{V}\left(\mathbf{r}, s_{1} s_{2}\right)$ defined in Eqs. (3.14) and (3.15).

The PC potential contains the operators $f_{M}(r), f_{M}^{S}(r)$
$\left(\boldsymbol{\sigma}_{\Lambda} \cdot \boldsymbol{\sigma}_{N}\right)$, and $f_{M}^{T}(r) S_{\Lambda N}(\hat{r})$, and the corresponding matrix elements read

$$
\begin{aligned}
& \left(p P l L \lambda S J\left|f_{M}(r)\right| 1 \mathrm{INL} \lambda^{\prime} S^{\prime} J\right) \\
& =\delta_{l \mid} \delta_{\lambda \lambda^{\prime}} \delta_{S S^{\prime}} \delta_{L \mathrm{~L}}(P L \mid \mathrm{NL})\left(p l\left|f_{M}(r)\right| 1 \mathrm{l}\right), \\
& \left(p P l L \lambda S J\left|f_{M}^{S}(r)\left(\boldsymbol{\sigma}_{\Lambda} \cdot \boldsymbol{\sigma}_{N}\right)\right| 1 \mathrm{INL} \lambda^{\prime} S^{\prime} J\right) \\
& =\delta_{l \mid} \delta_{\lambda \lambda^{\prime}} \delta_{S S^{\prime}} \delta_{L \mathrm{~L}}(P L \mid \mathrm{NL}) \\
& \quad \times\left(p l\left|f_{M}^{S}(r)\right| 1 \mathrm{l}\right)[2 S(S+1)-3],
\end{aligned}
$$

$$
\begin{aligned}
(p P l & \left.\lambda J\left|f_{M}^{T}(r) S_{\Lambda N}(\hat{r})\right| 1 \mathrm{INL} \lambda^{\prime} S^{\prime} J\right) \\
= & (-)^{L+l+J+1} \delta_{S S^{\prime}} \delta_{L L} \delta_{S 1} \sqrt{120} \hat{\lambda} \hat{\lambda}^{\prime} \hat{l}(P L \mid \mathrm{NL}) \\
& \times\left(p l\left|f_{M}^{T}(r)\right| 1 \mid\right)\left\{\begin{array}{rrr}
\lambda^{\prime} & 1 & J \\
1 & \lambda & 2
\end{array}\right\}\left\{\begin{array}{ccc}
l & \mid & 2 \\
\lambda^{\prime} & \lambda & L
\end{array}\right\} \\
& \times(l 020 \mid 10),
\end{aligned}
$$

with

$$
(P L \mid \mathrm{NL})=\delta_{L \mathrm{~L}} \int R^{2} d R_{j_{L}}(P R) \mathcal{R}_{\mathrm{NL}}(R)
$$

and

$$
\left(p l\left|f_{M}\right| 1 \mathrm{I}\right)=\int r^{2} d r j_{l}(p r) f_{M}(r) \mathcal{R}_{1 \mid}(r), \quad \text { etc. }
$$

The PV potentials are of the form

$$
\mathcal{V}^{\mathrm{PV}}\left(\mathbf{r}, s_{1}, s_{2}\right) \sim \mathbf{S} \cdot \mathbf{f}^{( \pm)}(r) \quad \text { with } \mathbf{S}=\left\{\begin{array}{l}
\boldsymbol{\sigma}_{\Lambda}, \\
\boldsymbol{\sigma}_{N}, \\
i \boldsymbol{\sigma}_{\Lambda} \times \boldsymbol{\sigma}_{N},
\end{array}\right.
$$

and we obtain

$$
\begin{aligned}
\left(p P l L l S J\left|\mathcal{V}_{M}^{P V}\right| \mathrm{nINL} l^{\prime} S^{\prime} J^{\prime}\right) \\
=\delta_{L L} \hat{l} \hat{l}^{\prime} \hat{\imath}(1010 \mid l 0)\left\{\begin{array}{lll}
l & \mathrm{~L} & l^{\prime} \\
l & 1 & l
\end{array}\right\}\left\{\begin{array}{ccc}
l^{\prime} & S^{\prime} & J \\
S & l & 1
\end{array}\right\} \\
\quad \times(-)^{J+S+l+L}\left\langle S\left\|\mathbf{S}_{M}\right\| S^{\prime}\right\rangle\left(p l\left|f_{M}^{( \pm)}\right| \mathrm{nl}\right)(P L \mid \mathrm{NL}) .
\end{aligned}
$$

The spin-dependent matrix elements are

$$
\begin{aligned}
\left\langle S\left\|\boldsymbol{\sigma}_{N}\right\| S^{\prime}\right\rangle & =\sqrt{6} \hat{S} \hat{S}^{\prime}(-)^{S}\left\{\begin{array}{ccc}
1 / 2 & 1 / 2 & S^{\prime} \\
1 & S & 1 / 2
\end{array}\right\} \\
& =(-)^{S+S^{\prime}}\left\langle S\left\|\boldsymbol{\sigma}_{\Lambda}\right\| S^{\prime}\right\rangle
\end{aligned}
$$

and 


$$
\begin{aligned}
\left\langle S\left\|i\left(\boldsymbol{\sigma}_{\Lambda} \times \boldsymbol{\sigma}_{N}\right)\right\| S^{\prime}\right\rangle & =\sqrt{12}\left(\delta_{S 0} \delta_{S^{\prime} 1}+\delta_{S 1} \delta_{S^{\prime} 0}\right) \\
& =-\left\langle S\left\|i\left(\boldsymbol{\sigma}_{N} \times \boldsymbol{\sigma}_{\Lambda}\right)\right\| S^{\prime}\right\rangle .
\end{aligned}
$$

The matrix elements $\left(p l\left|f_{M}^{( \pm)}\right| \mathrm{nl}\right)$ are easily evaluated, and one obtains

$$
\left(p l\left|f_{M}^{( \pm)}\right| \mathrm{nl}\right)=\left(p l\left|f_{M}^{L}\right| \mathrm{nl}\right) \pm\left(p l\left|f_{M}^{R}\right| \mathrm{nl}\right),
$$

with

$$
\begin{aligned}
\left(p l\left|f_{M}^{R}\right| \mathrm{nl}\right) \equiv & \int r^{2} d r j_{l}(p r) f_{M}(r) \\
& \times\left(\frac{1}{r} \frac{d}{d r} r+\frac{\mathrm{I}(\mathrm{I}+1)-l(l+1)}{2 r}\right) \mathcal{R}_{\mathrm{nl}}(r)
\end{aligned}
$$

and

$$
\begin{aligned}
\left(p l\left|f_{M}^{L}\right| \mathrm{nl}\right) \equiv & -\int r^{2} d r \mathcal{R}_{\mathrm{nl}}(r) f_{M}(r)\left(\frac{1}{r} \frac{d}{d r} r\right. \\
& \left.+\frac{l(l+1)-\mathrm{I}(\mathrm{I}+1)}{2 r}\right) j_{l}(p r) .
\end{aligned}
$$

Note that the "sum rule"

$$
\begin{aligned}
\left(p l\left|f_{M}^{L}\right| \mathrm{nl}\right)-\left(p l\left|f_{M}^{R}\right| \mathrm{nl}\right) & =\left(p l\left|f_{M}^{\prime}\right| \mathrm{nl}\right) \\
& =\int r^{2} d r j_{l}(p r) f_{M}^{\prime}(r) \mathcal{R}_{\mathrm{nl}}(r)
\end{aligned}
$$

should always be obeyed.

The radial integral (A10) can be expressed as

$$
\begin{aligned}
\left(p l\left|f_{M}^{L}\right| \mathrm{nl}\right)= & -p \int r^{2} d r \mathcal{R}_{\mathrm{nl}}(r) f_{M}(r) \\
& \times\left\{\frac{(l+2)(l+1)-\mathrm{I}(\mathrm{I}+1)}{2(2 l+1)} j_{l-1}(p r)\right. \\
& \left.+\frac{l(l-1)-\mathrm{I}(\mathrm{I}+1)}{2(2 l+1)} j_{l+1}(p r)\right\},
\end{aligned}
$$

which immediately leads to

$$
\left(p l\left|f_{M}^{L}\right| \mathrm{nl}\right)=\mp p \int r^{2} d r j_{\mathrm{I}}(p r) f_{M}(r) \mathcal{R}_{\mathrm{nl}}(r) \quad \text { for } l=\mathrm{I} \pm 1 .
$$

We are interested here only in

$$
\mathcal{R}_{1 l}=\left(\pi b^{2}\right)^{-1 / 4} \sqrt{\frac{l !}{(2 l+1) !}}\left(\frac{2}{b}\right)^{l+1} r^{l} \exp \left(-\frac{r^{2}}{2 b^{2}}\right),
$$

and, in order to simplify the integral (A9), the following relationship can be used:

$$
\frac{1}{r} \frac{d}{d r} r \mathcal{R}_{1 l}=\left(\frac{l+1}{r}-\frac{r}{b^{2}}\right) \mathcal{R}_{1 l}
$$

We obtain

$$
\left(p l\left|f_{M}^{R}\right| 1 \mathrm{I}\right)=-\frac{1}{\mathrm{~b}^{2}}\left\{\begin{array}{c}
\int r^{3} d r j_{l}(p r) f_{M}(r) \mathcal{R}_{1 \mid}(r) \\
\text { for } l=\mathrm{I}+1, \\
\int\left(r^{2}-\hat{\mathrm{I}}^{2} \mathrm{~b}^{2}\right) r d r j_{l}(p r) f_{M}(r) \mathcal{R}_{1 \mid}(r) \\
\text { for } l=\mathrm{I}-1 .
\end{array}\right.
$$

It should be remembered that the radial wave functions $\mathcal{R}_{\mathrm{nl}}(r)$ and $\mathcal{R}_{\mathrm{NL}}(R)$ have to be evaluated with harmonic oscillator parameters $\mathrm{b}=\sqrt{2} b$ and $\mathrm{b}=b / \sqrt{2}$, respectively, $\mathrm{b}$ being the oscillator length for the harmonic mean-field potential.

As indicated in Eq. (5.1) the FNS effects are incorporated directly into the radial integrals through the replacements $f_{M}(r) \rightarrow \overline{f_{M}}(r)$, etc. At variance, the SRCs, given by Eq. (5.2), are added by the substitutions

$\left.|1 \mathrm{Im}\rangle \rightarrow \overline{|1 \mathrm{Im}\rangle}=g_{i}(r)|1| \mathrm{Im}\right\rangle, \quad|p l m\rangle \rightarrow \overline{|p l m\rangle}=g_{f}(r)|p l m\rangle$,

in Eqs. (A1) and (A3), and when the FNSs and SRCs are included simultaneously, the radial integrals (A3) become

$$
\overline{\left(p l\left|f_{M}\right| 1 \mathrm{I}\right)}=\int r^{2} d r j_{l}(p r) g_{f}(r) \overline{f_{M}}(r) g_{i}(r) \mathcal{R}_{1 \mid}(r) .
$$

Thus it is equivalent to comprise the SRCs either through the wave functions, as done in Eq. (A17), or by renormalizing the radial form factor $\overline{f_{M}}(r) \rightarrow g_{f}(r) \overline{f_{M}}(r) g_{i}(r)$. The same is valid for $f_{M}^{\prime}(r), f_{M}^{S}(r)$, and $f_{M}^{T}(r)$. On the contrary, for the integrals (A9) and (A10), which contain derivatives, from Eq. (A17) one has

$$
\begin{aligned}
& \overline{\left(p l\left|f_{M}^{L}\right| 1 \mathrm{I}\right)}=-\int r^{2} d r\left[j_{l}(p r) g_{f}^{\prime}(r) \pm p j_{\mathrm{I}}(p r) g_{f}(r)\right] \overline{f_{M}}(r) g_{i}(r) \mathcal{R}_{11}(r) \quad \text { for } l=\mathrm{I} \pm 1, \\
& \overline{\left(p l\left|f_{M}^{R}\right| 1 \mid\right)}= \begin{cases}\int r^{2} d r j_{l}(p r) g_{f}(r) \overline{f_{M}}(r)\left[g_{i}^{\prime}(r)-r \mathrm{~b}^{-2} g_{i}(r)\right] \mathcal{R}_{1 \mid}(r) & \text { for } l=\mathrm{I}+1, \\
\int r^{2} d r j_{l}(p r) g_{f}(r) \overline{f_{M}}(r)\left[g_{i}^{\prime}(r)-\left(r \mathrm{~b}^{-2}-\hat{\imath}^{2} r^{-1}\right) g_{i}(r)\right] \mathcal{R}_{1 \mid}(r) & \text { for } l=\mathrm{I}-1,\end{cases}
\end{aligned}
$$


being $g^{\prime}(r) \equiv d g(r) / d r$. In this case it is no longer possible to include the SRCs via the form factor, which is a direct consequence of the fundamental difference between the FNS effects and SRCs. Namely, while the SRCs modify the nuclear wave functions, the FNS renormalization is done directly on the vertices of the Feynman diagrams that determine the one-meson-exchange transition potential.

Finally, the isospin matrix elements needed in the calculation are

$$
\begin{gathered}
\left\langle 1,-1\left|\mathcal{T}_{0}\right|-\frac{1}{2},-\frac{1}{2}\right\rangle=1, \quad\left\langle 1,-1\left|\mathcal{T}_{1}\right|-\frac{1}{2},-\frac{1}{2}\right\rangle=1, \\
\left\langle 0,0\left|\mathcal{T}_{0}\right|-\frac{1}{2}, \frac{1}{2}\right\rangle=-\frac{1}{\sqrt{2}}, \quad\left\langle 1,0\left|\mathcal{T}_{0}\right|-\frac{1}{2}, \frac{1}{2}\right\rangle=\frac{1}{\sqrt{2}}, \\
\left\langle 0,0\left|\mathcal{T}_{1}\right|-\frac{1}{2}, \frac{1}{2}\right\rangle=\frac{3}{\sqrt{2}}, \quad\left\langle 1,0\left|\mathcal{T}_{1}\right|-\frac{1}{2}, \frac{1}{2}\right\rangle=\frac{1}{\sqrt{2}} .
\end{gathered}
$$

[1] H. Park et al., Phys. Rev. C 61, 054004 (2000).

[2] M. M. Block and R. H. Dalitz, Phys. Rev. Lett. 11, 96 (1963).

[3] J. B. Adams, Phys. Rev. 156, 1611 (1967).

[4] B. H. J. McKellar and B. F. Gibson, Phys. Rev. C 30, 322 (1984).

[5] K. Takeuchi, H. Takaki, and H. Bando, Prog. Theor. Phys. 73, 841 (1985).

[6] J. Cohen, Prog. Part. Nucl. Phys. 25, 139 (1990).

[7] W. M. Alberico, A. De Pace, M. Ericson, and A. Molinari, Phys. Lett. B 256, 134 (1991).

[8] A. Ramos, E. van Meijgaard, C. Bennhold, and B. K. Jennings, Nucl. Phys. A644, 703 (1992).

[9] A. Ramos, E. Oset, and L. L. Salcedo, Phys. Rev. C 50, 2314 (1994).

[10] A. Parreño, A. Ramos, and E. Oset, Phys. Rev. C 51, 2477 (1995).

[11] A. Parreño, A. Ramos, and C. Bennhold, Phys. Rev. C 52, R1768 (1995); 54, 500(E) (1996).

[12] J. F. Dubach, G. B. Feldman, B. R. Holstein, and L. de la Torre, Ann. Phys. (N.Y.) 249, 146 (1996).

[13] A. Parreño, A. Ramos, and C. Bennhold, Phys. Rev. C 56, 339 (1997).

[14] A. Ramos, M. J. Vicente-Vacas, and E. Oset, Phys. Rev. C 55, 735 (1997).

[15] E. Oset and A. Ramos, Prog. Part. Nucl. Phys. 41, 191 (1998).

[16] A. Parreño, A. Ramos, C. Bennhold, and K. Maltman, Phys. Lett. B 435, 1 (1998).

[17] T. Inoue, M. Oka, T. Motoba, and K. Itonaga, Nucl. Phys. A633, 312 (1998).

[18] K. Itonaga, T. Ueda, and T. Motoba, Nucl. Phys. A639, 329c (1998)

[19] A. Parreño, A. Ramos, N. G. Kelkar, and C. Bennhold, Phys. Rev. C 59, 2122 (1999).

[20] W. M. Alberico and G. Garbarino, Phys. Lett. B 486, 362 (2000).

[21] K. Sasaki, T. Inoue, and M. Oka, Nucl. Phys. A669, 331 (2000); A678, 455(E) (2000).

[22] K. Sasaki, T. Inoue, and M. Oka, Nucl. Phys. A678, 455 (2000).

[23] W. M. Alberico, A. De Pace, G. Garbarino, and A. Ramos, Phys. Rev. C 61, 044314 (2000).

[24] A. Parreño and A. Ramos, Phys. Rev. C 65, 015204 (2002); A. Parreño, A. Ramos, and C. Bennhold, ibid. 65, 015205 (2002).

[25] E. Oset, D. Jido, and J. E. Palomar, Nucl. Phys. A691, 146 (2001); D. Jido, E. Oset, and J. E. Palomar, ibid. A694, 525 (2001).
[26] J.-H. Jun, Phys. Rev. C 63, 044012 (2001).

[27] K. Itonaga, T. Ueda, and T. Motoba, Phys. Rev. C 65, 034617 (2002).

[28] M. N. Nagels, T. A. Rijken, and J. J. de Swart, Phys. Rev. D 15, 2547 (1977).

[29] P. M. M. Maessen, Th. A. Rijken, and J. J. de Swart, Phys. Rev. C 40, 2226 (1989).

[30] D. Halderson, Phys. Rev. C 48, 581 (1993).

[31] A. Parreño, A. Ramos, C. Bennhold, and D. Halderson, Dynamical Features of Nuclei and Finite Fermi Systems (World Scientific, Singapore, 1994), p. 318.

[32] B. Desplanques, J. Donoghue, and B. R. Holstein, Ann. Phys. (N.Y.) 124, 449 (1980).

[33] R. E. Marshak, Riazuddin, and C. P. Ryan, Theory of Weak Interactions in Particle Physics (Wiley Interscience, New York, 1969).

[34] L. de la Torre, Ph.D. thesis, University of Massachusetts, 1982.

[35] L. B. Okun, Leptons and Quarks (North-Holland, Amsterdam, 1982).

[36] E. D. Commins and P. H. Bucksbaum, Weak Interactions of Leptons and Quarks (Cambridge University Press, Cambridge, England, 1983).

[37] G. Nardulli, Phys. Rev. C 38, 832 (1988).

[38] G. E. Brown and M. Rho, Phys. Rep. 363, 85 (2002).

[39] A. Montwill et al., Nucl. Phys. A234, 413 (1974).

[40] J. J. Szymanski et al., Phys. Rev. C 43, 849 (1991).

[41] H. Noumi et al., Phys. Rev. C 52, 2936 (1995).

[42] H. Bhang et al., Phys. Rev. Lett. 81, 4321 (1998).

[43] O. Hashimoto et al., Phys. Rev. Lett. 88, 042503 (2002).

[44] E. G. Adelberger and W. C. Haxton, Annu. Rev. Nucl. Part. Sci. 35, 501 (1985).

[45] W. Haeberli and B. R. Holstein, in Symmetries and Fundamental Interactions in Nuclei, edited by W. C. Haxton and E. M. Henley (World Scientific, Singapore, 1995).

[46] W. C. Haxton and C. E. Wieman, Annu. Rev. Nucl. Part. Sci. 51, 261 (2001).

[47] J. Golak, H. Kamada, K. Miyagama, H. Witala, and W. Glöckle, Phys. Rev. Lett. 83, 3142 (1999).

[48] P. Ring and P. Schuck, The Nuclear Many Body Problem (Springer-Verlag, New York, 1980).

[49] A. Bohr and B. R. Mottelson, Nuclear Structure (Benjamin, New York, 1969), Vol. I.

[50] M. Moshinsky, Nucl. Phys. 13, 104 (1959).

[51] F. Krmpotić, A. Mariano, and A. Samana, Phys. Lett. B 541, 298 (2002) 